Discrete Comput Geom 33:321-344 (2005)

DOI: $10.1007 /$ s00454-004-1154-y

\title{
Canonical Ordering Trees and Their Applications in Graph Drawing*
}

\author{
Huaming Zhang and Xin He \\ Department of Computer Science and Engineering, \\ State University of New York at Buffalo, \\ Buffalo, NY 14260, USA \\ \{huazhang,xinhe\}@cse.buffalo.edu
}

\begin{abstract}
We study the properties of Schnyder's realizers and canonical ordering trees of plane graphs. Based on these newly discovered properties, we obtain compact drawings of two styles for any plane graph $G$ with $n$ vertices. First, we show that $G$ has a visibility representation with height at most $\lceil 15 n / 16\rceil$. This improves the previous best bound of $(n-1)$. Second, we show that every plane graph $G$ has a straight-line grid embedding on an $\left(n-\delta_{0}-1\right) \times\left(n-\delta_{0}-1\right)$ grid, where $\delta_{0}$ is the number of cyclic faces of $G$ with respect to its minimum realizer. This improves the previous best bound of $(n-1) \times(n-1)$.

We also study the properties of the regular edge labeling of 4-connected plane triangulation. Based on these properties, we show that every such a graph has a canonical ordering tree with at most $\lceil(n+1) / 2\rceil$ leaves. This improves the previously known bound of $\lfloor(2 n+1) / 3\rfloor$. We show that every 4 -connected plane graph has a visibility representation with height at most $\lceil 3 n / 4\rceil$. All drawings discussed in this paper can be obtained in linear time.
\end{abstract}

\section{Introduction}

The concepts of canonical ordering and canonical ordering tree of plane triangulations and triconnected plane graphs played crucial roles in designing several graph-drawing algorithms [8], [10], [11], [14], [16]-[18]. Recently, Chiang et al. generalized these concepts to arbitrary connected plane graphs [5], which leads to improvements in several graph-drawing algorithms [4], [5].

A visibility representation (VR for short) of a plane graph $G$ is a representation, where the vertices of $G$ are represented by nonoverlapping horizontal segments (called vertex segments), and each edge of $G$ is represented by a vertical line segment touching the vertex segments of its end vertices. The problem of computing a compact VR is

\footnotetext{
* This research was supported in part by NSF Grant CCR-9912418 and NSF Grant CCR-0309953.
} 
important not only in algorithmic graph theory, but also in practical applications such as VLSI layout. A VR of a 2-connected plane graph $G$ can be obtained from an stnumbering of $G$ and the corresponding st-numbering of its dual [26], [29]. Using this approach, the height of the VR is bounded by $(n-1)$ and the width of the VR is bounded by $(2 n-5)$ [26], [29].

Some work have been done to reduce the width of the VR. Kant and He showed that every 4-connected plane graph has a VR with width at most $(n-1)$ [19]. Kant proved that every plane graph has a VR with width at most $\lfloor(3 n-6) / 2\rfloor[18]$. Very recently, Lin et al. reduced the width to $\lfloor(22 n-42) / 15\rfloor$ by choosing the best $s t$-numbering from three $s t$-numberings derived from a Schnyder's realizer of $G$ [23]. However, the height of the VR of a general plane graph remains to be the trivial bound of $(n-1)$. In this paper we prove that every plane graph $G$ has a VR with height at most $\lceil 15 n / 16\rceil$.

Finding a straight-line grid embedding of a plane graph $G$ is another extensively studied problem. It has been known for long time that such an embedding exists. However, the drawings produced by earlier algorithms require grids of exponential size [10]. A breakthrough was achieved in [7], [10], and [11]: It was shown that such an embedding can be done on a $(2 n-4) \times(n-2)$ grid. The grid size was reduced to $(n-1) \times(n-1)$ in [28]. For a 4-connected plane triangulation $G$ with at least four exterior vertices, the size of the grid can be reduced to $(n / 2-1) \times(n / 2)$ [13], [24], which is optimal in the sense that there are an infinite number of 4-connected plane graphs, any grid drawings of which need rectangular grids of width $(n / 2-1)$ and height $n / 2$. Chrobak and Kant [6] dealt with convex grid drawings of 3-connected planar graphs. It is known that there exists a plane graph whose straight-line embedding requires a grid of size at least $\lceil 2 n / 3\rceil \times\lceil 2 n / 3\rceil$. It has been conjectured that every plane graph $G$ has such an embedding on a $\lceil 2 n / 3\rceil \times\lceil 2 n / 3\rceil$ grid. However, the best known bound remains $(n-1) \times(n-1)$ given in [28]. In this paper we show that every plane graph has a straight-line grid embedding on an $\left(n-\delta_{0}-1\right) \times\left(n-\delta_{0}-1\right)$ grid, where $\delta_{0}$ is the number of clockwise cyclic faces of $G$ with respect to its minimum realizer $\mathcal{R}_{0}$. Our work is the first result reducing the grid size below $(n-1)$ by a nontrivial parameter.

In many applications of a canonical ordering tree $T$, the number of leaves of $T$ is a crucial parameter. For example, in the floor-planning algorithm in [4] and the 2-visibility drawing algorithm in [5] for plane graphs, the width of both drawings are bounded by the number of leaves of the canonical ordering tree used. Thus, the problems of finding more compact drawings are reduced to finding a canonical ordering tree with fewer leaves. However, it is known that there exists a plane graph for which every canonical ordering tree has at least $\lfloor(2 n+1) / 3\rfloor$ leaves. Thus the width of the drawings produced by the algorithms in [4] and [5] is bounded by $\lfloor(2 n+1) / 3\rfloor$.

We study properties of regular edge labeling (REL for short) of a 4-connected plane triangulation $G$. The properties and applications of REL have been studied in [1], [12], [19], and [20]. Our study reveals the interconnections between the REL, the canonical ordering tree and Schnyder's realizer. This leads to the finding of a canonical ordering tree with at most $\lceil(n+1) / 2\rceil$ leaves for a 4-connected plane triangulation. Based on this, we prove that every 4-connected plane graph $G$ has a VR with height at most $\lceil 3 n / 4\rceil$. All drawings discussed in this paper can be obtained in linear time.

Our drawing methods are somewhat different from the previous ones. For the VR of a plane graph, we not only rely on the existence of special canonical ordering trees with a 
certain number of leaves, but also introduce a new way of obtaining st-numbering from a canonical ordering tree, namely, we alternately assign numbers to tree nodes from left to right or from right to left, and so on. Hopefully, this technique can be further used to improve the height bound for VR, or even the width bound for VR if we consider the canonical ordering tree for the dual graph. As the straight-line grid embedding is concerned, we use the minimum realizer instead of the general Schnyder's realizer to obtain more compact embedding in most practical cases.

The present paper is organized as follows. Section 2 introduces preliminaries. Section 3 gathers concepts and results related to canonical ordering trees, which are interesting by themselves and are needed in later sections. Section 4 presents the construction of a VR with height at most $\lceil 15 n / 16\rceil$. In Section 5 we present the algorithm for compact straightline grid embedding. Section 6 explores the properties of REL of a 4-connected plane triangulation $G$ and proves $G$ has a canonical ordering tree with at most $\lceil(n+1) / 2\rceil$ leaves. Section 7 studies the connection between the REL and the $s t$-numbering and proves a 4-connected plane graph has a VR with height at most $\lceil 3 n / 4\rceil$. Section 8 concludes the paper.

\section{Preliminaries}

In this section we give definitions and preliminary results. $G=(V, E)$ denotes a graph with $n=|V|$ vertices and $m=|E|$ edges. The degree $\operatorname{deg}(v)$ of a vertex $v$ is the number of edges incident to $v$. A planar graph $G$ is a graph which can be embedded on the plane without edge crossings. A plane graph is a planar graph with a fixed embedding. An embedding of a plane graph divides the plane into a number of regions, called faces. The unbounded region is the exterior face. Other regions are interior faces. The vertices and the edges on the exterior face are called exterior vertices and exterior edges. Other vertices and edges are interior vertices and interior edges. A path $P$ of $G$ is a sequence of distinct vertices $u_{1}, u_{2}, \ldots, u_{k}$ such that $\left(u_{i}, u_{i+1}\right) \in E$ for $1 \leq i<k$. We also use $P$ to denote the set of the edges in it. Each $u_{i}$ for $1<i<k$ is called an internal vertex of $P$. Furthermore, if $\left(u_{k}, u_{1}\right) \in E$, then $u_{1}, u_{2}, \ldots, u_{k}$ is called a cycle. We normally use $C$ to denote a cycle and the set of the edges in it. If $C$ contains $k$ vertices, it is a $k$-cycle. A triangle (quadrangle, resp.) is a 3-cycle (4-cycle, resp.) A cycle $C$ of $G$ divides the plane into its interior and exterior regions. If $C$ contains at least one vertex in its interior region, $C$ is called a separating cycle. If all facial cycles of $G$ are triangles, $G$ is a plane triangulation. A graph $G$ is 2-connected if for any vertex $u$ in $G, G-\{u\}$ is connected. $G$ is 4-connected if for any three distinct vertices $u_{1}, u_{2}, u_{3}$ in $G, G-\left\{u_{1}, u_{2}, u_{3}\right\}$ is connected. A 4-connected plane triangulation does not have separating triangles except the boundary cycle of its exterior face. $G$ is called a directed graph (digraph for short) if each edge of $G$ is assigned a direction. We abbreviate the words "counterclockwise" and "clockwise" as ccw and cw, respectively.

The dual graph $G^{*}=\left(V^{*}, E^{*}\right)$ of a plane graph $G$ is defined as follows: For each face $F$ of $G, G^{*}$ has a vertex $v_{F}$. For each edge $e$ in $G, G^{*}$ has a dual edge $e^{*}=\left(v_{F_{1}}, v_{F_{2}}\right)$ where $F_{1}$ and $F_{2}$ are the two faces of $G$ with $e$ on their common boundaries.

Let $G$ be a 2-connected plane digraph with two specified exterior vertices $s$ and $t$. $G$ is called an st-plane graph if it is acyclic with $s$ as the only source and $t$ as the only 
sink. The properties of $s t$-plane graphs were studied in [21] and [26]. In particular, for every face $f$ of $G$, its boundary cycle consists of two directed paths. The path on its left (right, resp.) side is called the left (right, resp.) path of $f$. There is exact one source (sink, resp.) vertex on the boundary of $f$, it is called the source (sink, resp.) of $f$.

An orientation of a graph $G$ is a digraph obtained from $G$ by assigning a direction to each edge of $G$. We use $G$ to denote both the resulting digraph and the underlying undirected graph unless otherwise specified. (Its meaning will be clear from the context.) For a plane graph $G$ and two vertices $s, t$, an orientation of $G$ is called an st-orientation if the resulting digraph is an $s t$-plane graph.

Let $G$ be a 2-connected plane graph and let $s, t$ be two distinct exterior vertices of $G$. An st-numbering of $G$ is a one-to-one mapping $\xi: V \rightarrow\{1,2, \ldots, n\}$, such that $\xi(s)=$ $1, \xi(t)=n$, and each vertex $v \neq s, t$ has two neighbors $u, w$ with $\xi(u)<\xi(v)<\xi(w)$, where $u$ ( $w$, resp.) is called a smaller neighbor (bigger neighbor, resp.) of $v$. Given an $s t$-numbering $\xi$ of $G$, we can orient $G$ by directing each edge in $E$ from its lower numbered end vertex to its higher numbered end vertex. The resulting orientation is called the orientation derived from $\xi$ which, obviously, is an $s t$-orientation of $G$. On the other hand, if $G=(V, E)$ has an $s t$-orientation $\mathcal{O}$, we can define a one-to-one mapping $\xi: V \rightarrow\{1, \ldots, n\}$ by topological sort. It is easy to see that $\xi$ is an $s t$-numbering and the orientation derived from $\xi$ is $\mathcal{O}$.

Lempel et al. [21] showed that for every 2-connected plane graph $G$ and any two exterior vertices $s$ and $t$, there exists an $s t$-numbering. The following lemma was given in [26] and [29]:

Lemma 1. Let $G$ be a 2-connected plane graph. Let $\xi$ be an st-numbering of $G$. A $V R$ of $G$ can be obtained from $\xi$ in linear time. The height of the VR is the length of the longest directed path in the st-orientation of $G$ derived from $\xi$.

\section{Canonical Ordering Tree and Orderly Spanning Tree}

Let $G$ be a plane triangulation with $n \geq 3$ vertices and $m=3 n-6$ edges. Let $v_{1}, v_{2}, \ldots, v_{n}$ be an ordering of the vertices of $G$ where $v_{1}, v_{2}, v_{n}$ are the three exterior vertices of $G$ in ccw order. Let $G_{k}$ be the subgraph of $G$ induced by $v_{1}, v_{2}, \ldots, v_{k}$ and let $H_{k}$ be the exterior face of $G_{k}$. Let $G-G_{k}$ be the subgraph of $G$ obtained by removing $v_{1}, v_{2}, \ldots, v_{k}$.

Definition 1. [11] An ordering $v_{1}, \ldots, v_{n}$ of a plane triangulation $G$ is canonical if the following hold for every $k=3, \ldots, n$ :

(1) $G_{k}$ is biconnected, and its exterior face $H_{k}$ is a cycle containing the edge $\left(v_{1}, v_{2}\right)$.

(2) The vertex $v_{k}$ is on the exterior face of $G_{k}$, and its neighbors in $G_{k-1}$ form a subinterval of the path $H_{k-1}-\left(v_{1}, v_{2}\right)$ with at least two vertices. Furthermore, if $k<n, v_{k}$ has at least one neighbor in $G-G_{k}$. (Note that the case $k=3$ is degenerated, and $H_{2}-\left(v_{1}, v_{2}\right)$ is regarded as the edge $\left(v_{1}, v_{2}\right)$ itself.)

Figure 1 illustrates a canonical ordering of a plane triangulation. A canonical ordering of $G$ can be viewed as an order in which $G$ is reconstructed from a single edge $\left(v_{1}, v_{2}\right)$ 


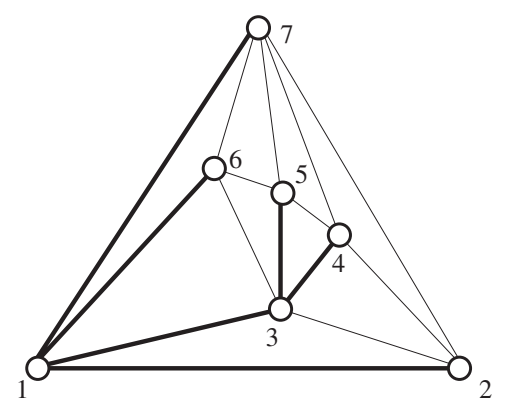

Fig. 1. A canonical ordering tree of a plane triangulation $G$.

step by step. At step $k$, when $v_{k}$ is added to construct $G_{k}$, let $c_{l}, c_{l+1}, \ldots, c_{r}$ be the lower ordered neighbors of $v_{k}$ from left to right on the exterior face of $G_{k-1}$. We call $\left(v_{k}, c_{l}\right)$ the left edge of $v_{k},\left(v_{k}, c_{r}\right)$ the right edge of $v_{k}$, and the edges $\left(c_{p}, v_{k}\right)$ with $l<p<r$ the internal edges of $v_{k}$. The collection $T$ of the left edges of the vertices $v_{j}$ for $3 \leq j \leq n$ plus the edge $\left(v_{1}, v_{2}\right)$ is a spanning tree of $G$ and is called a canonical ordering tree of $G$ [11], [15]. The tree drawn in thick lines in Fig. 1 is a canonical ordering tree of $G$.

Let $T$ be a rooted spanning tree of a plane graph $G$. Two distinct vertices of $G$ are unrelated with respect to $T$ if neither of them is an ancestor of the other in $T$. An edge of $G$ is unrelated with respect to $T$ if its end vertices are unrelated. While traveling $T$ in ccw (cw, resp.) preorder (postorder, resp.), if each vertex of $G$ is assigned a number from $\{1,2, \ldots, n\}$ according to the order being visited, the resulting order is called the $c c w$ ( $c w$, resp.) preordering (postordering, resp.) of $G$ with respect to $T$.

In [5] the concept of a canonical ordering tree was generalized to any connected plane graph, which Chiang et al. call an orderly spanning tree, as follows:

Definition 2. Let $G$ be a plane graph and let $T$ be a spanning tree of $G$. Let $v_{1}, v_{2}, \ldots, v_{n}$ be the ccw preordering of the vertices of $G$ with respect to $T$.

(1) A vertex $v_{i}$ of $G$ is orderly with respect to $T$ if the neighbors of $v_{i}$ in $G$ form the following four blocks in ccw order around $v_{i}$ :

$B_{1}(v i)$ : the parent of $v_{i}$,

$B_{2}(v i)$ : the unrelated neighbors $v_{j}$ of $v_{i}$ with $j<i$,

$B_{3}(v i)$ : the children of $v_{i}$, and

$B_{4}(v i)$ : the unrelated neighbors $v_{j}$ of $v_{i}$ with $j>i$, where each block could be empty.

(2) $T$ is called an orderly spanning tree of $G$ if $v_{1}$ is an exterior vertex, and each $v_{i}(1 \leq i \leq n)$ is orderly with respect to $T$.

Let $T$ be an orderly spanning tree of a plane triangulation $G$. Let $v_{1}, v_{2}, \ldots, v_{n}$ be the ccw preordering of the vertices of $G$ with respect to $T$. It is easy to see that $v_{1}, v_{2}, v_{n}$ are the exterior vertices in ccw order, and for any interior vertex $v_{i}(3 \leq i \leq n-1)$, both $B_{2}\left(v_{i}\right)$ and $B_{4}\left(v_{i}\right)$ are nonempty. 


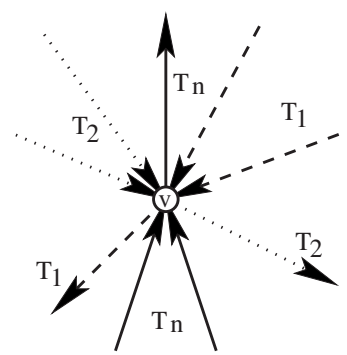

Fig. 2. Edge directions around an interior vertex $v$.

The following is another related concept called Schnyder's realizer [27], [28]:

Definition 3. Let $G$ be a plane triangulation with three exterior vertices $v_{1}, v_{2}, v_{n}$ in ccw order. A realizer $\mathcal{R}$ of $G$ is a partition of the interior edges of $G$ into three sets $T_{1}, T_{2}, T_{n}$ of directed edges such that the following hold:

- For each $i \in\{1,2, n\}$, the interior edges incident to $v_{i}$ are in $T_{i}$ and directed toward $v_{i}$.

- For each interior vertex $v$ of $G, v$ has exactly one edge leaving $v$ in each of $T_{1}, T_{2}, T_{n}$. The ccw order of the edges incident to $v$ is: leaving in $T_{1}$, entering in $T_{n}$, leaving in $T_{2}$, entering in $T_{1}$, leaving in $T_{n}$ and entering in $T_{2}$ (see Fig. 2). Each entering block could be empty.

It was shown in [27] and [28] that every plane triangulation $G$ has a realizer $\mathcal{R}$, and each $T_{i}(i \in\{1,2, n\})$ is a tree rooted at the vertex $v_{i}$ containing all interior vertices of $G$. Figure 3 shows a realizer of a plane triangulation $G$. Three trees $T_{1}, T_{2}, T_{n}$ are drawn

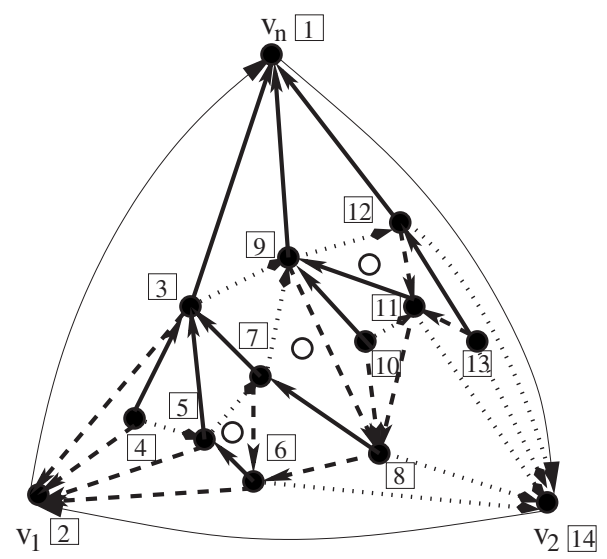

Fig. 3. A plane triangulation $G$ and the minimum realizer of $G$. 
as dashed lines, dotted lines, and thick solid lines, respectively. (Ignore the small boxes containing integers and the directions of the exterior edges for now. Their meaning will be explained later.)

The following lemma summarizes the results related to the above concepts. They were shown in [4], [9], [22], [23], [25], and [27]:

Lemma 2. Let $G$ be a plane triangulation with three exterior vertices $v_{1}, v_{2}, v_{n}$ in ccw order.

(1) If $T$ is an orderly spanning tree of $G$, then the ccw preordering of the vertices of $G$ with respect to $T$ is a canonical ordering of $G$. Hence $T$ is also a canonical ordering tree of $G$. A canonical ordering tree of $G$ is also an orderly spanning tree of $G$.

(2) Let $\left\{T_{1}, T_{2}, T_{n}\right\}$ be a realizer of $G$, where $T_{i}$ is rooted at $v_{i}$ for each $i=1,2, n$. Then each $T_{i}$ plus both exterior edges of $G$ incident to $v_{i}$ is an orderly spanning tree of $G$.

(3) A canonical ordering tree $T$ of $G$ with at most $\lfloor(2 n+1) / 3\rfloor$ leaves is obtainable in $O(n)$ time. There is a plane triangulation $G$ for which every canonical ordering tree has at least $\lfloor(2 n+1) / 3\rfloor$ leaves.

(4) A realizer of $G$ can be obtained from an orderly spanning tree $T$ in $O(n)$ time, where one of the trees in the realizer is obtained from $T$ by removing the two edges on the exterior face.

The following lemma shows the connection between canonical ordering trees and st-numberings.

Lemma 3. Let $G$ be a plane triangulation and let $T$ be a canonical ordering tree (or, equivalently, an orderly spanning tree) of $G$ rooted at $v_{1}$. Then the ccw preordering $v_{1}, v_{2}, \ldots, v_{n}$ of the vertices of $G$ with respect to $T$ is an st-numbering of $G$.

Proof. First observe that $v_{1}, v_{2}, v_{n}$ are the exterior vertices of $G$ in ccw order. For $v_{1}$ and $v_{2}, v_{n}$ is their bigger neighbor. For $v_{2}$ and $v_{n}, v_{1}$ is their smaller neighbor. For any other vertex $v_{i}, 3 \leq i \leq n-1$, both $B_{2}\left(v_{i}\right)$ and $B_{4}\left(v_{i}\right)$ are nonempty. The parent of $v_{i}$ in $T$ and all vertices in $B_{2}\left(v_{i}\right)$ are smaller neighbors of $v_{i}$. The children of $v_{i}$ (if any) in $T$ and all vertices in $B_{4}\left(v_{i}\right)$ are bigger neighbors of $v_{i}$.

For example, consider the tree $T_{n}$ (rooted at $v_{n}$ ) shown in Fig. 3. By Lemma 2(1) and (2), the union of $T_{n}$ and the two exterior edges $\left(v_{1}, v_{n}\right)$ and $\left(v_{2}, v_{n}\right)$ is a canonical ordering tree of $G$. Denote it by $T_{n}^{\prime}$. The ccw preordering of the vertices of $G$ with respect to $T_{n}^{\prime}$ are shown in integers inside the small boxes. It is an $s t$-numbering of $G$ by Lemma 3.

Let $G$ be a plane triangulation with three exterior vertices $v_{1}, v_{2}, v_{n}$ in ccw order. Let $\mathcal{R}=\left\{T_{1}, T_{2}, T_{n}\right\}$ be a realizer of $G$. We direct the exterior edges as $v_{1} \rightarrow v_{n}, v_{n} \rightarrow$ $v_{2}, v_{2} \rightarrow v_{1}$. The resulting orientation is called the orientation induced by $\mathcal{R}$ and denoted by $G(\mathcal{R})$. Note that $G(\mathcal{R})$ is never an $s t$-orientation because it is always cyclic.

Let $\delta(\mathcal{R})$ denote the number of interior cyclic faces of $G(\mathcal{R})$. Let $\eta_{1}, \eta_{2}, \eta_{n}$ be the number of internal (i.e. nonleaf) vertices in $T_{1}, T_{2}, T_{n}$, respectively. The following results were proved in [2], [3], [16], and [23]: 
Lemma 4. Let $G$ be a plane triangulation with three exterior vertices $v_{1}, v_{2}, v_{n}$ in ccw order.

(1) Let $\mathcal{R}=\left\{T_{1}, T_{2}, T_{n}\right\}$ be any realizer of $G$. Then $\eta_{1}+\eta_{2}+\eta_{n}-\delta(\mathcal{R})=n-1$.

(2) There is an unique realizer $\mathcal{R}_{0}$ of $G$ such that all interior cyclic faces of $G\left(\mathcal{R}_{0}\right)$ are directed in cw direction. $\mathcal{R}_{0}$ can be obtained in $O(n)$ time.

The realizer $\mathcal{R}_{0}$ stated in statement (2) of Lemma 4 is called the minimum realizer of $G$ [2], [3]. We use $\mathcal{R}_{0}$ to construct a canonical ordering tree with at least $\lceil(n+1) / 2\rceil$ leaves, which in turn enables us to find more compact VR representation. We also use $\mathcal{R}_{0}$ to construct a more compact straight-line grid embedding later.

For example, the realizer shown in Fig. 3 is actually the minimum realizer $\mathcal{R}_{0}$ of $G$. The orientation of $G$ drawn in the figure is the orientation of $G\left(\mathcal{R}_{0}\right)$. It has three cw interior cyclic faces, marked by empty circles. We call such faces the cyclic faces of $G\left(\mathcal{R}_{0}\right)$. (Note that although the exterior face is cyclic in $G\left(\mathcal{R}_{0}\right)$, we do not count it in $\delta\left(\mathcal{R}_{0}\right)$ since it is not an interior face.)

In the induced orientation $G\left(\mathcal{R}_{0}\right)$ :

- $\mathcal{F}_{3 \mathrm{cw}}$ denotes the set of interior faces of $G\left(\mathcal{R}_{0}\right)$ with three cw edges and $\delta_{0}=\left|\mathcal{F}_{3 \mathrm{cw}}\right|$.

- $\mathcal{F}_{2 \mathrm{cw}}$ denotes the set of interior faces of $G\left(\mathcal{R}_{0}\right)$ with two cw edges and one ccw edge and $\alpha_{0}=\left|\mathcal{F}_{2 \mathrm{cw}}\right|$.

- $\mathcal{F}_{1 \mathrm{cw}}$ denotes the set of interior faces of $G\left(\mathcal{R}_{0}\right)$ with one cw edge and two ccw edges and $\beta_{0}=\left|\mathcal{F}_{1 \mathrm{cw}}\right|$.

Note that $\mathcal{F}_{3 \mathrm{cw}}, \mathcal{F}_{2 \mathrm{cw}}, \mathcal{F}_{1 \mathrm{cw}}$ form a partition of the set of the interior faces of $G\left(\mathcal{R}_{0}\right)$. The following theorem is needed in later sections.

\section{Theorem 1.}

(1) $\delta_{0}+\alpha_{0}=n-\delta_{0}-1$.

(2) $\delta_{0} \leq\lfloor(n-1) / 2\rfloor$.

(3) $G$ has a canonical ordering tree with at least $\lceil(n+1) / 2\rceil$ leaves, which can be obtained in linear time.

Proof. (1) The number of interior faces of $G\left(\mathcal{R}_{0}\right)$ is $\delta_{0}+\alpha_{0}+\beta_{0}=2 n-5$. Since every edge (including the exterior edges) of $G\left(\mathcal{R}_{0}\right)$ is a cw edge of exactly one interior face, we have $3 \delta_{0}+2 \alpha_{0}+\beta_{0}=3 n-6$. This gives $2 \delta_{0}+\alpha_{0}=n-1$, which implies (1).

(2) Since $2 \delta_{0}=n-\alpha_{0}-1 \leq n-1$, we have $\delta_{0} \leq\lfloor(n-1) / 2\rfloor$.

(3) Let $T_{i}^{\prime}(i \in\{1,2, n\})$ be the canonical ordering tree obtained by adding the two exterior edges incident to $v_{i}$ to $T_{i}$. Let $\eta_{i}^{\prime}$ be the number of internal vertices of the tree $T_{i}^{\prime}$, which obviously is the same as the number of the internal vertices of $T_{i}$. By Lemma 4(1), we have $\eta_{1}^{\prime}+\eta_{2}^{\prime}+\eta_{n}^{\prime}-\delta_{0}=n-1$. Let $l_{i}(i \in\{1,2, n\})$ be the number of leaves of $T_{i}^{\prime}$. Then $\eta_{i}{ }^{\prime}=n-l_{i}$. Thus $l_{1}+l_{2}+l_{n}=2 n+1-\delta_{0}$. Because $\delta_{0} \leq\lfloor(n-1) / 2\rfloor$, we have $l_{1}+l_{2}+l_{n} \geq\lceil(3 n+3) / 2\rceil$. Hence at least one of $l_{i} \geq\lceil(n+1) / 2\rceil$.

Note, it takes linear time to construct the minimum realizer $\mathcal{R}_{0}$ [3]. Thus, the construction of such a canonical ordering tree can be obtained in linear time. 
For a 4-connected plane triangulation $G$, there exists a canonical ordering tree $T$ of $G$ with at most $\lceil(n+1) / 2\rceil$ leaves. Since we need to use a different technique and the proof is lengthy, we put it in the separate section, Section 6.

\section{Compact Visibility Representation with Reduced Width}

In this section we present our theorem on the height of the compact VR. Here, the compactness is meant to be only in one dimension: the height. Let $G$ be a plane triangulation with exterior vertices $v_{1}, v_{2}, v_{n}$ in ccw order. Let $T$ be an orderly spanning tree of $G$ with at least $\lceil(n+1) / 2\rceil$ leaves, and let $T$ be rooted at $v_{n}$. Let $\rho$ be the ccw preordering of the vertices of $G$ with respect to $T$. In this ordering, $\rho\left(v_{n}\right)=1, \rho\left(v_{1}\right)=2$ and $\rho\left(v_{2}\right)=n$. For any vertex $v$ other than $v_{1}, v_{2}, v_{n}, v$ has a nonempty set $B_{2}(v)$ of smaller neighbors and a nonempty set $B_{4}(v)$ of bigger neighbors.

We construct two vertex numberings of $G$ according to $T$ simultaneously. The first vertex numbering $\xi_{T}$ of $G$ is defined as follows:

Step 1: Traveling from the leftmost unassigned leaf of $T$ by ccw postordering with respect to $T$. (The first visited vertex is $v_{1}$.) Stop assigning numbers when we reach either the next leaf of $T$ or the root $v_{n}$. When we reach $v_{n}$, the numbering process is complete. When we reach a leaf, do not assign a number to it at this moment. Continue to step 2 if there are leaves remaining to be traveled.

Step 2: Traveling from the rightmost unassigned leaf of $T$ by $\mathbf{c w}$ postordering with respect to $T$. (Initially, it is $v_{2}$.) Stop assigning numbers when we reach either the next leaf of $T$ or the root $v_{n}$. When we reach $v_{n}$, we are done. When we reach a leaf, do not assign a number to it at this moment. Loop back to step 1 if there are leaves remaining to be traveled.

Figure 4(a) illustrates this numbering process using the canonical ordering tree $T=T_{n}^{\prime}$ (shown as thick solid lines), which is obtained from the minimum realizer in Fig. 3 by applying Lemma 2(2). The integers inside the small boxes are the numbers assigned to the vertices. In this process we visit $v_{1}$ first in step 1 , then move to step 2 to visit $v_{2}$. Then we loop back to step 1 to visit the vertex numbered 3, followed by step 2 to visit vertices numbered 4 and 5, and so on, until we reach $v_{n}$. Note that we always have $\xi_{T}\left(v_{1}\right)=1$, $\xi_{T}\left(v_{2}\right)=2$ and $\xi_{T}\left(v_{n}\right)=n$.

The second vertex numbering $\xi_{T}^{\prime}$ is defined similarly, except that the order of steps 1 and 2 are swapped. Figure 4(b) shows the numbering $\xi_{T}^{\prime}$ for the graph $G$ in Fig. 3 by using the canonical ordering tree $T_{n}^{\prime}$. Note that we always have $\xi_{T}^{\prime}\left(v_{2}\right)=1, \xi_{T}^{\prime}\left(v_{1}\right)=2$ and $\xi_{T}^{\prime}\left(v_{n}\right)=n$.

Next we prove the following technical lemma:

Lemma 5. Let $T$ be a canonical ordering tree of a plane triangulation $G$. Then $\xi_{T}$, $\xi_{T}^{\prime}$ are two st-numberings of $G$. The st-orientation of $G$ derived from $\xi_{T}$ ( $\xi_{T}^{\prime}$, resp.) are denoted by $G_{T}\left(G_{T}^{\prime}\right.$, resp.).

Proof. We show that $\xi_{T}$ is an st-numbering of $G$. For each vertex other than the root $v_{n}$, its parent in $T$ is assigned a bigger number by $\xi_{T}$. For each vertex $v$ other than $v_{1}$, 


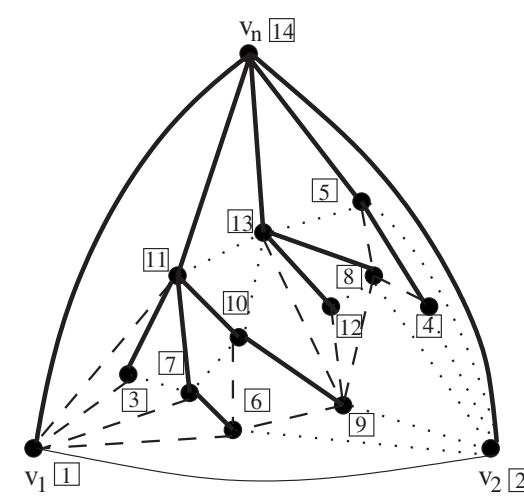

(a)

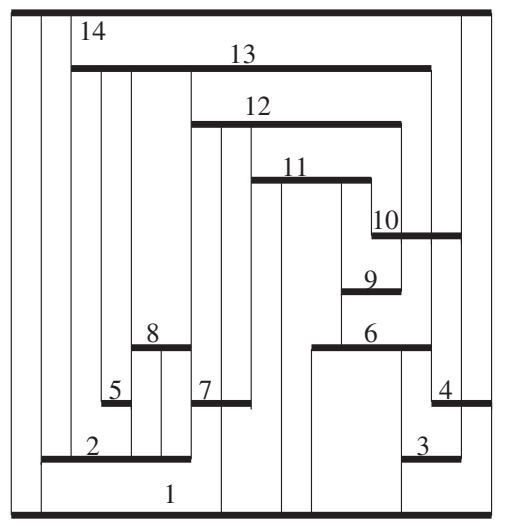

(c)

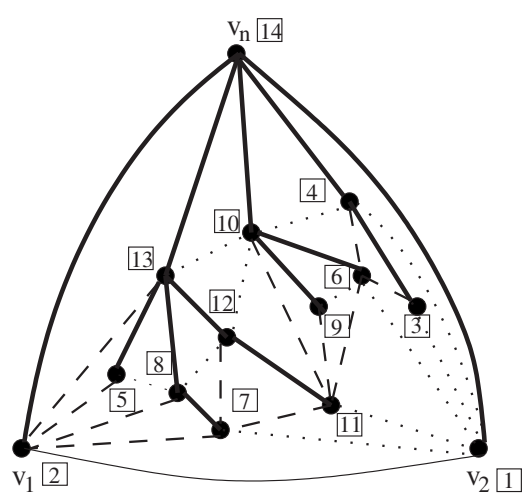

(b)

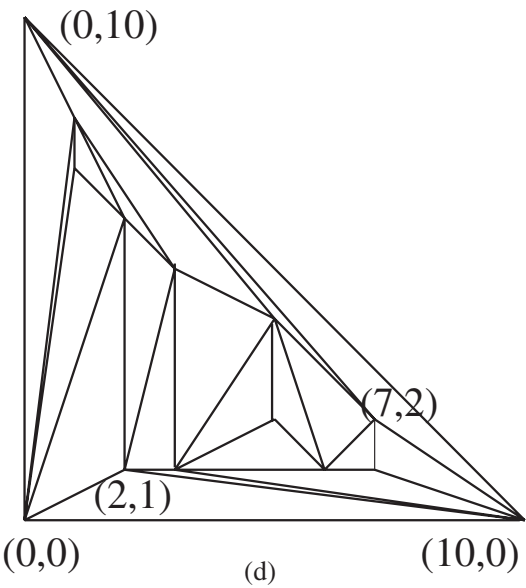

Fig. 4. Two drawings of the graph $G$ in Fig. 3.

we need to show that at least one of its neighbors is assigned a smaller number by $\xi_{T}$. There are four cases.

Case 1: $v$ is not a leaf of $T$. Its children in $T$ are smaller neighbors of $v$.

Case 2: $v$ is $v_{2} . \quad v_{1}$ is its smaller neighbor.

Case 3: $v$ is a leaf other than $v_{1}$ and is numbered in step 1. All the vertices in the nonempty set $B_{2}(v)$ are traveled before $v$ by $\xi_{T}$. Hence, they are smaller neighbors of $v$.

Case 4: $v$ is a leaf other than $v_{2}$ and is numbered in step 2. All the vertices in the nonempty set $B_{4}(v)$ are traveled before $v$ by $\xi_{T}$. Hence, they are smaller neighbors of $v$.

Therefore, $\xi_{T}$ is an st-numbering. Similarly, we can show $\xi_{T}^{\prime}$ is also an $s t$-numbering. 


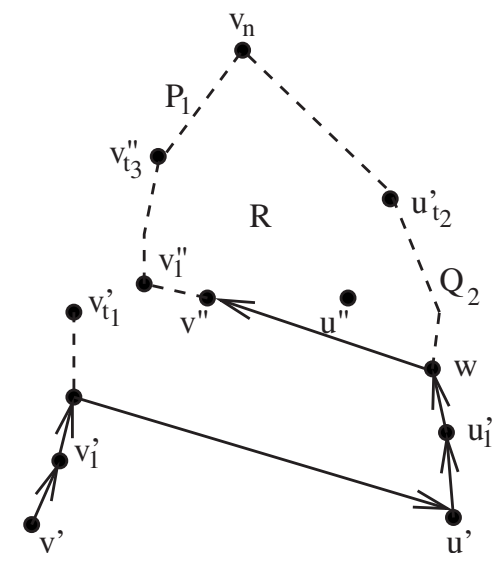

Fig. 5. The proof of Lemma 6 .

Let $v^{\prime}, v^{\prime \prime}\left(u^{\prime}, u^{\prime \prime}\right.$, resp.) be the $(2 i-1)$ th and $2 i$ th leaf traveled by $\xi_{T}$ in step 1 (step 2 , resp.). Then $u^{\prime}, u^{\prime \prime}\left(v^{\prime}, v^{\prime \prime}\right.$, resp.) are the $(2 i-1)$ th and $2 i$ th leaf traveled by $\xi_{T}^{\prime}$ in its own step 1 (step 2, resp.). These four leaves are called the $i$ th block of leaves of $T$. For example, in Fig. 4(b), the vertices $v^{\prime}=2, v^{\prime \prime}=5, u^{\prime}=1, u^{\prime \prime}=3$ are the first block of leaves of $T_{n}^{\prime}$. The order of the four leaves in the $i$ th block traveled by $\xi_{T}\left(\xi_{T}^{\prime}\right.$, resp.) is $v^{\prime}, u^{\prime}, v^{\prime \prime}, u^{\prime \prime}\left(u^{\prime}, v^{\prime}, u^{\prime \prime}, v^{\prime \prime}\right.$, resp.). Though it is possible that some nonleaf vertices of $T$ are traveled by $\xi_{T}\left(\xi_{T}^{\prime}\right.$, resp.) between the four leaves.

Lemma 6. Let $T$ be a canonical ordering tree of a plane triangulation $G$. Let $P$ be a directed path in $G_{T}$, and let $P^{\prime}$ be a directed path in $G_{T}^{\prime}$. Then for any block $B$ of leaves of $T$, one of $P$ and $P^{\prime}$ cannot pass through all four vertices in $B$.

Proof. If $P$ does not pass through all four vertices in $B$, we are done. Let us assume that $P$ passes through all four vertices in $B$. Let $v_{1}^{\prime}, \ldots, v_{t_{1}}^{\prime}$ (can be degenerated to empty set) be all the vertices traveled by $\xi_{T}$ between $v^{\prime}$ and $u^{\prime}$. (See Fig. 5 for an illustration. Some edges on $P$ are drawn in solid lines.) Then $v_{1}^{\prime}, \ldots, v_{t_{1}}^{\prime}$ are all the vertices traveled between $v^{\prime}$ and $u^{\prime \prime}$ by $\xi_{T}^{\prime}$. Let $u_{1}^{\prime}, \ldots, u_{t_{2}}^{\prime}$ (can be an empty set) be all the vertices traveled by $\xi_{T}$ between $u^{\prime}$ and $v^{\prime \prime}$. Then $u^{\prime}, u_{1}^{\prime}, \ldots, u_{t_{2}}^{\prime}$ are on the unique path, denoted by $Q_{1}$, of $T$ from $u^{\prime}$ to the root $v_{n}$. Let $v_{1}^{\prime \prime}, \ldots, v_{t_{3}}^{\prime \prime}$ (can be an empty set) be all the vertices traveled by $\xi_{T}$ between $v^{\prime \prime}$ and $u^{\prime \prime}$. Then $v^{\prime \prime}, v_{1}^{\prime \prime}, \ldots, v_{t_{3}}^{\prime \prime}$ are on the unique path of $T$ from $v^{\prime \prime}$ to the root $v_{n}$, denote this unique path by $P_{1}$.

Since $P$ passes through both $u^{\prime}$ and $v^{\prime \prime}$, and $u_{1}^{\prime}, \ldots, u_{t_{2}}^{\prime}$ are all the vertices traveled by $\xi_{T}$ between $u^{\prime}$ and $v^{\prime \prime}$, there is a vertex $w \in\left\{u^{\prime}, u_{1}^{\prime}, \ldots, u_{t_{2}}^{\prime}\right\}$ such that the edge $\left(w, v^{\prime \prime}\right)$ is in $P$, and $\xi_{T}\left(v^{\prime \prime}\right)>\xi_{T}(w) \geq \xi_{T}\left(u^{\prime}\right)$. Denote the unique path from $w$ to $v_{n}$ in $T$ by $Q_{2}$. Note that $Q_{2}$ is a subpath of $Q_{1}$.

Let $R$ be the closed region enclosed by the edge $\left(w, v^{\prime \prime}\right)$ and the paths $P_{1}$ and $Q_{2}$. Now consider the position of $u^{\prime \prime}$ with respect to $R$. Because $Q_{1}$ is the path connecting $u^{\prime}$ to the root $v_{n}$ in $T$ and $u^{\prime \prime}$ is a leaf of $T, u^{\prime \prime}$ cannot be on $Q_{1}$. Therefore, $u^{\prime \prime}$ cannot be on $Q_{2}$. Similarly, $u^{\prime \prime}$ cannot be on $P_{1}$. So $u^{\prime \prime}$ is either inside or outside of $R$. 
Suppose $u^{\prime \prime}$ is outside of $R$. Consider the unique path in $T$ from $u^{\prime \prime}$ to the root $v_{n}$. There are two possibilities: (1) It intersects with $P_{1}$ from the left. Then $\xi_{T}$ travels $u^{\prime \prime}$ before $v^{\prime \prime}$, which is impossible. (2) It intersects with $Q_{2}$ from the right. Then $\xi_{T}$ travels $u^{\prime \prime}$ before $u^{\prime}$, which is again impossible. Therefore, $u^{\prime \prime}$ must be inside $R$.

Similarly, it can be shown that $v^{\prime}$ and all $v_{j}^{\prime}\left(1 \leq j \leq t_{1}\right)$ are outside of $R$. Therefore none of $v^{\prime}$ or $v_{j}^{\prime}\left(1 \leq j \leq t_{1}\right)$ can be a neighbor of $u^{\prime \prime}$.

Consider the path $P^{\prime}$. If $v^{\prime}$ is not on $P^{\prime}$, we are done. Assume $v^{\prime}$ is on $P^{\prime}$. If $P^{\prime}$ passes through $u^{\prime \prime}$ after it passes through $v^{\prime}$, it has to reach $u^{\prime \prime}$ from one of $v^{\prime}, v_{1}^{\prime}, \ldots, v_{t_{1}}^{\prime}$. (This is because $v_{1}^{\prime}, \ldots, v_{t_{1}}^{\prime}$ are all the vertices traveled between $v^{\prime}$ and $u^{\prime \prime}$ by $\xi_{T}^{\prime}$.) However, since none of $v^{\prime}, v_{j}^{\prime}, 1 \leq j \leq t_{1}$, is a neighbor of $u^{\prime \prime}$, this is impossible. Thus $P^{\prime}$ cannot pass through all four vertices in $B$.

Theorem 2. Every plane graph $G$ with $n$ vertices has a VR with height at most $\lceil 15 n / 16\rceil$, which can be obtained in linear time.

Proof. Without loss of generality, we assume $G$ is a plane triangulation. By Theorem 1(3), from the minimal realizer $\mathcal{R}_{0}$ of $G$, we can obtain a canonical ordering tree $T$ of $G$ with at least $l \geq\lceil(n+1) / 2\rceil$ leaves. Thus $T$ has at least $\lfloor n / 8\rfloor$ disjoint blocks of leaves. $T$ induces two $s t$-orientations $G_{T}$ and $G_{T}^{\prime}$ by Lemma 5 . This can be done in $O(n)$ time.

Let $P$ be a longest directed path in $G_{T}$, and let $P^{\prime}$ be a longest directed path in $G_{T}^{\prime}$. By Lemma 6, for any block $B$ of leaves of $T$, one of $P$ or $P^{\prime}$ has to bypass at least one leaf in $B$. Thus $P$ and $P^{\prime}$ together have to bypass at least $\lfloor n / 8\rfloor$ leaves. So one of them has to bypass at least $\lfloor n / 16\rfloor$ leaves. Hence its length is at most $\lceil 15 n / 16\rceil$. Thus the length of the longest directed path in one of $G_{T}, G_{T}^{\prime}$ is at most $\lceil 15 n / 1\rceil$.

We apply the VR algorithm in Lemma 1 to one of $G_{T}, G_{T}^{\prime}$ whose longest directed path is shorter. This results in a VR of $G$ with height at most $\lceil 15 n / 16\rceil$.

First, it takes linear time to obtain $T$ from $G$ by Theorem 1. Obviously, the construction of the $s t$-numbering of $G$ based on $T$ as described above takes linear time. Applying Lemma 1, the total running time of constructing the VR is $O(n)$.

For example, in Fig. 4(b), the longest directed path in the orientation derived from the given $s t$-numbering passes through the vertices numbered 1, 3, 4, 6, 9,10, 11, 12, 13, 14 . However, in the $s t$-numbering shown in Fig. 3, the longest directed path passes through all vertices. Figure 4(c) shows the VR of the graph $G$, using the $s t$-numbering in Fig. 4(b).

\section{Compact Straight-Line Grid Embedding}

In this section we use the minimum realizer $\mathcal{R}_{0}$ of a plane triangulation $G$ to present our theorem on the size of a compact straight-line grid embedding.

Define an order relation $<_{\text {lex }}$ on two pairs of numbers $\left(x_{1}, x_{2}\right)$ and $\left(y_{1}, y_{2}\right)$ as follows: $\left(x_{1}, x_{2}\right)<_{\operatorname{lex}}\left(y_{1}, y_{2}\right)$ iff either $x_{1}<y_{1}$, or $x_{1}=y_{1}$ and $x_{2}<y_{2}$. The following definition and lemma were given in [27] and [28]. 
Definition 4. A weak barycentric representation of a graph $G=(V, E)$ is an injective mapping $\pi: v \in V \rightarrow\left(\pi_{1}(v), \pi_{2}(v), \pi_{n}(v)\right) \in R^{3}$ that satisfies the following conditions:

(1) $\pi_{1}(v)+\pi_{2}(v)+\pi_{n}(v)=1$ for all vertices $v$.

(2) For each edge $(x, y) \in E$ and each vertex $z \notin\{x, y\}$, there is a $k \in\{1,2, n\}$ such that $\left(\pi_{k}(x), \pi_{k-1}(x)\right)<_{\operatorname{lex}}\left(\pi_{k}(z), \pi_{k-1}(z)\right)$ and $\left(\pi_{k}(y), \pi_{k-1}(y)\right)<\operatorname{lex}$ $\left(\pi_{k}(z), \pi_{k-1}(z)\right)$ (when $k=1$ or $n, k-1$ denotes $n$ or 2 , resp.).

Lemma 7. Let $\pi: v \in V \rightarrow\left(\pi_{1}(v), \pi_{2}(v), \pi_{n}(v)\right)$ be a weak barycentric representation of $G$. Then given any noncolinear points $\mathbf{a}, \mathbf{b}, \mathbf{c}$ on the plane, the mapping $f: v \in V \rightarrow \pi_{1}(v) \mathbf{a}+\pi_{2}(v) \mathbf{b}+\pi_{n}(v) \mathbf{c}$ defines a straight-line embedding of $G$ in the plane spanned by $\mathbf{a}, \mathbf{b}, \mathbf{c}$.

Let $G=(V, E)$ be a plane graph with three exterior vertices $v_{1}, v_{2}, v_{n}$ in ccw order. Let $\mathcal{R}_{0}=\left\{T_{1}, T_{2}, T_{n}\right\}$ be the minimum realizer of $G$. Let $\mathcal{F}=\mathcal{F}_{3 \mathrm{cw}} \cup \mathcal{F}_{2 \mathrm{cw}}$ be the set of interior faces of $G\left(\mathcal{R}_{0}\right)$ that have either three or two cw edges on their boundaries. Thus $|\mathcal{F}|=\delta_{0}+\alpha_{0}$.

For each vertex $v \in G$, let $P_{1}(v)\left(P_{2}(v)\right.$ and $P_{3}(v)$, resp.) be the path in $T_{1}\left(T_{2}\right.$ and $T_{n}$, resp.) from $v$ to the root $v_{1}\left(v_{2}\right.$ and $v_{n}$, resp.). $P_{1}(v), P_{2}(v), P_{n}(v)$ divides $G$ into three regions $R_{1}(v), R_{2}(v)$ and $R_{n}(v)$, where $R_{i}(v)$ denotes the closed region bounded by the paths $P_{j}(v), P_{k}(v)$ and the exterior edge $\left(v_{j}, v_{k}\right)(i \neq j, k)$.

For each interior vertex $v$ of $G$, let $\pi_{1}(v)\left(\pi_{2}(v)\right.$ and $\pi_{n}(v)$, resp.) be the number of faces of $G\left(\mathcal{R}_{0}\right)$ in $R_{1}(v)\left(R_{2}(v)\right.$ and $R_{n}(v)$, resp.) that belong to $\mathcal{F}$. Note that since the two interior faces of $G\left(\mathcal{R}_{0}\right)$ incident to the exterior edges $\left(v_{1}, v_{2}\right)$ and $\left(v_{1}, v_{n}\right)$ are not in $R_{1}(v)$ and these two faces belong to $\mathcal{F}$, we have $\pi_{1}(v) \leq \delta_{0}+\alpha_{0}-2$. Similarly, we have $\pi_{2}(v), \pi_{n}(v) \leq \delta_{0}+\alpha_{0}-2$.

For the three exterior vertices $v_{1}, v_{2}$ and $v_{n}$, define $\pi_{i}\left(v_{i}\right)=\delta_{0}+\alpha_{0}$ and $\pi_{j}\left(v_{i}\right)=0$ for $j \neq i$. Note that for all vertices $v, \pi_{1}(v)+\pi_{2}(v)+\pi_{n}(v)=\delta_{0}+\alpha_{0}$.

Lemma 8. If $u \in R_{i}(v)-P_{i+1}(v)$, then $\pi_{i}(u)<\pi_{i}(v)$ (when $i=2$ or $n, i+1$ denotes $n$ or 1 , resp.).

Proof. We only prove the case $i=n$. The other cases are symmetric. We need to show that $u \in R_{n}(v)-P_{1}(v)$ implies $\pi_{n}(u)<\pi_{n}(v)$.

Case 1: $u$ is in the interior of the region $R_{n}(v)$. Let $Q$ be the path in $T_{n}$ from $u$ to the root $v_{n}$ of $T_{n} . Q$ must intersect either $P_{1}(v)$ or $P_{2}(v)$ at a vertex $p$.

Case 1(a): Suppose $p$ is on $P_{2}(v)$. Let $r$ be the parent of $p$ in $T_{2}$. Let $(q, p)$ be the edge incident to $p$ that is next to the edge $(r, p)$ in cw order around $p$. (See Fig. 6(a).) Let $f$ be the face of $G$ bounded by the edges $(p, r),(r, q),(q, p)$. Note that $f$ is in $R_{n}(v)$ but not in $R_{n}(u)$. By the property of the realizer, the edge $(q, p)$ must be in $T_{n}$ and directed toward $p$. The edge $(p, r)$ is in $T_{2}$ and directed toward $r$. So $f$ has at least two cw edges on its boundary, and hence is in $\mathcal{F}$. Thus, $\pi_{n}(u) \leq \pi_{n}(v)-1<\pi_{n}(v)$. 


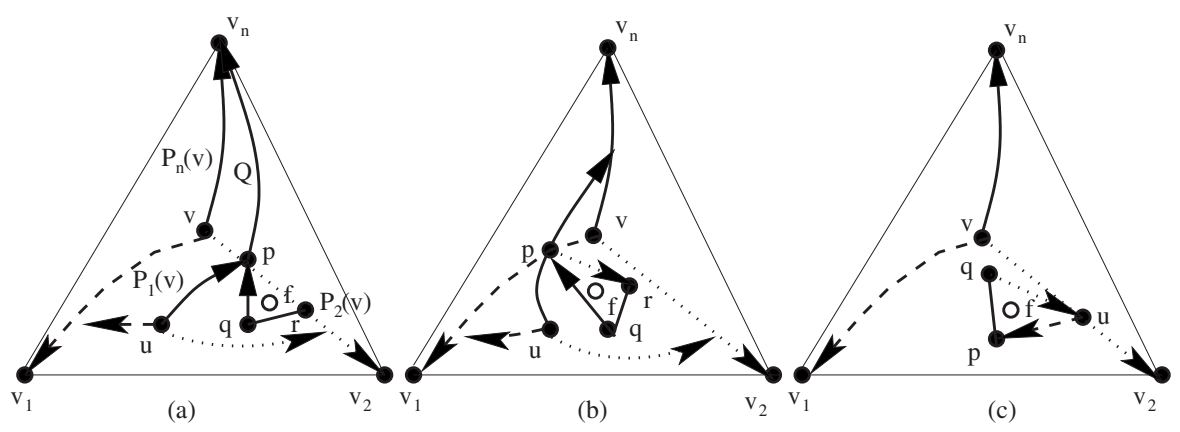

Fig. 6. The proof of Lemma 8.

Case 1(b): Suppose $p$ is on $P_{1}(v)$ (but $\left.p \neq v\right)$. By the property of the realizer, there must be an edge $(p, r)$ in $T_{2}$ directed from $p$ to $r$ and this edge must be in the region $R_{n}(v)$. (See Fig. 6(b).) Let $(q, p)$ be the edge incident to $p$ that is next to the edge $(r, p)$ in cw order around $p$. Let $f$ be the face of $G$ bounded by the edges $(p, r),(r, q),(q, p)$. Note that $f$ is in $R_{n}(v)$ but not in $R_{n}(u)$. By the property of the realizer, the edge $(q, p)$ must be in $T_{n}$ and directed toward $p$. The edge $(p, r)$ is in $T_{2}$ and directed toward $r$. So $f$ has at least two cw edges on its boundary, and hence is in $\mathcal{F}$. Thus, $\pi_{n}(u) \leq \pi_{n}(v)-1<\pi_{n}(v)$.

Case 2: $u$ is on the path $P_{2}(v)$. By the property of the realizer, there exists an edge $(u, p)$ in $T_{1}$ directed toward $p$ and this edge must be in the region $R_{n}(v)$. Let $(u, q)$ be the edge incident to $u$ that is next to the edge $(u, p)$ in cw order around $u$. Let $f$ be the face bounded by the edges $(u, p),(p, q),(q, u)$. (See Fig. 6(c).) Note that $f$ is in $R_{n}(v)$ but not in $R_{n}(u)$. By the property of the realizer, the edge $(q, u)$ must be in $T_{2}$ and directed toward $u$. The edge $(u, p)$ is in $T_{1}$ and directed toward $p$. So $f$ has at least two $\mathrm{cw}$ edges on its boundary and hence is in $\mathcal{F}$. Thus, $\pi_{n}(u) \leq \pi_{n}(v)-1<\pi_{n}(v)$.

Lemma 9. Let $u$ and $v$ be two distinct vertices of $G$. If $v$ is an interior vertex of $G$ and $u \in R_{i}(v)$, then $\left(\pi_{i}(u), \pi_{i-1}(u)\right)<_{\operatorname{lex}}\left(\pi_{i}(v), \pi_{i-1}(v)\right)$ (when $i=1$ or $n, i-1$ denotes $n$ or 2 , resp.).

Proof. We only prove the case $u \in R_{n}(v)$. The other cases are symmetric. We need to show $\left(\pi_{n}(u), \pi_{2}(u)\right)<_{\operatorname{lex}}\left(\pi_{n}(v), \pi_{2}(v)\right)$.

If $u \in R_{n}(v)-P_{1}(v)$, then by Lemma 8 we have $\pi_{n}(u)<\pi_{n}(v)$, which implies $\left(\pi_{n}(u), \pi_{2}(u)\right)<_{\text {lex }}\left(\pi_{n}(v), \pi_{2}(v)\right)$. Otherwise $u \in P_{1}(v)$. This implies $u \in R_{2}(v)-$ $P_{n}(v)$. By Lemma 8 with $i=2$, we have $\pi_{2}(u)<\pi_{2}(v)$. Since $R_{n}(u) \subset R_{n}(v)$, we have $\pi_{n}(u) \leq \pi_{n}(v)$. Thus $\left(\pi_{n}(u), \pi_{2}(u)\right)<_{\text {lex }}\left(\pi_{n}(v), \pi_{2}(v)\right)$ as was to be shown.

Theorem 3. The mapping $\pi: v \in V \rightarrow\left(1 /\left(\delta_{0}+\alpha_{0}\right)\right)\left(\pi_{1}(v), \pi_{2}(v), \pi_{n}(v)\right)$ is a weak barycentric representation.

Proof. The first condition of the barycentric representation is clearly satisfied, which implies the injectivity of $\pi$. We need to verify the second condition in Definition 4. 
Consider an edge $(x, y)$ of $G$ and a vertex $z \neq x, y$. If $z$ is an exterior vertex $v_{i}$, then $\pi_{i}(z)=\delta_{0}+\alpha_{0}>\pi_{i}(x), \pi_{i}(y)$, which implies $\left(\pi_{i}(x), \pi_{i-1}(x)\right)<_{\operatorname{lex}}\left(\pi_{i}(z), \pi_{i-1}(z)\right)$ and $\left(\pi_{i}(y), \pi_{i-1}(y)\right)<_{\operatorname{lex}}\left(\pi_{i}(z), \pi_{i-1}(z)\right)$.

Otherwise, $z$ is an interior vertex and $x, y \in R_{i}(z)$ for some $i \in\{1,2, n\}$. By Lemma 9 , we have $\left(\pi_{i}(x), \pi_{i-1}(x)\right)<_{\operatorname{lex}}\left(\pi_{i}(z), \pi_{i-1}(z)\right)$ and $\left(\pi_{i}(y), \pi_{i-1}(y)\right)<_{\operatorname{lex}}$ $\left(\pi_{i}(z), \pi_{i-1}(z)\right)$.

Theorem 4. Every plane triangulation $G$ with $n$ vertices has a straight-line grid embedding on a grid of size $\left(n-\delta_{0}-1\right) \times\left(n-\delta_{0}-1\right)$ where $\delta_{0}$ is the number of cyclic faces of $G$ with respect to its minimum realizer $\mathcal{R}_{0}$. The embedding can be constructed in linear time.

Proof. Pick three points $\mathbf{a}=(0,0), \mathbf{b}=\left(\delta_{0}+\alpha_{0}, 0\right)$ and $\mathbf{c}=\left(0, \delta_{0}+\alpha_{0}\right)$. By Theorem 3 and Lemma 7 , the mapping $v \rightarrow\left(\pi_{2}(v), \pi_{n}(v)\right)$ is a straight-line grid embedding on a grid of size $\left(\delta_{0}+\alpha_{0}\right) \times\left(\delta_{0}+\alpha_{0}\right)$. By Theorem 1(1), this equals the size stated in the theorem.

To construct the embedding, we need to construct the minimal realizer $\mathcal{R}_{0}$ of $G$, which can be done in linear time [3]. The calculation of the coordinates of the embedding can be done in $O(n)$ time by using the techniques given in [28].

As an example, Fig. 4(d) shows a straight-line grid embedding of $G$ on a grid of size $\left(n-\delta_{0}-1\right) \times\left(n-\delta_{0}-1\right)$. It is obtained by using the minimal realizer $\mathcal{R}_{0}$ of $G$ in Fig. 3 . For example, the coordinate of the vertex numbered 6 in Fig. 3 is $\left(\pi_{2}(6), \pi_{n}(6)\right)=(2,1)$.

\section{Canonical Ordering Tree with Fewer Leaves for 4-Connected Plane Triangulations}

In order to obtain a canonical ordering tree for a 4-connected plane triangulation with fewer leaves, we need another concept and a related lemma from [12] as follows:

Definition 5. Let $G^{\prime}$ be a plane graph with four vertices $v_{W}, v_{S}, v_{E}, v_{N}$ in ccw order on its exterior face. A regular edge labeling (REL for short) of $G^{\prime}$ is a partition of the interior edges of $G^{\prime}$ into two subsets $S_{1}, S_{2}$ of directed edges such that the following hold:

(1) For each interior vertex $v$, the edges incident to $v$ appear in ccw order around $v$ as follows: a set of edges in $S_{1}$ leaving $v$; a set of edges in $S_{2}$ entering $v$; a set of edges in $S_{1}$ entering $v$; a set of edges in $S_{2}$ leaving $v$. Each set is nonempty.

(2) All interior edges incident to $v_{N}$ are in $S_{1}$ and entering $v_{N}$. All interior edges incident to $v_{W}$ are in $S_{2}$ and leaving $v_{W}$. All interior edges incident to $v_{S}$ are in $S_{1}$ and leaving $v_{S}$. All interior edges incident to $v_{E}$ are in $S_{2}$ and entering $v_{E}$. Each block is not empty.

Lemma 10. Let $G^{\prime}$ be a plane graph with four vertices on its exterior face. $G^{\prime}$ has an REL if and only if the following conditions hold: (1) every interior face of $G^{\prime}$ is a triangle and the exterior face of $G^{\prime}$ is a quadrangle; (2) $G^{\prime}$ has no separating triangles. 


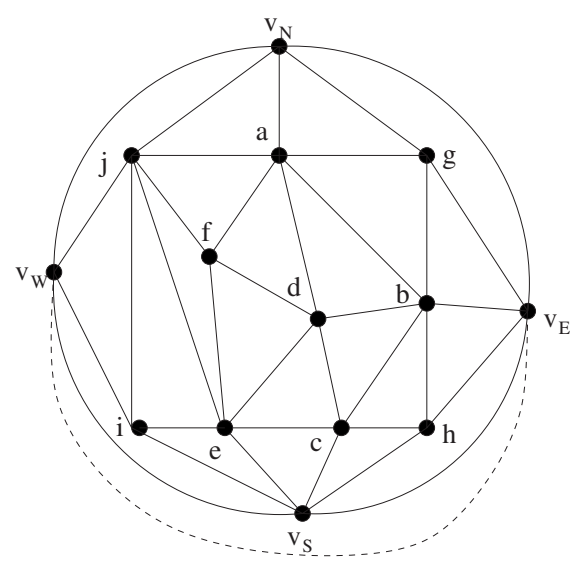

Fig. 7. A 4-connected plane triangulation $G$ and the PTP graph $G^{\prime}$ after deleting $\left(v_{\mathrm{W}}, v_{\mathrm{E}}\right)$.

A graph satisfying the two conditions in the above lemma is called a proper triangulated plane (PTP for short) graph.

Let $G$ be a 4-connected plane triangulation with three exterior vertices $v_{\mathrm{W}}, v_{\mathrm{E}}, v_{\mathrm{N}}$ in ccw order. Delete the edge $\left(v_{\mathrm{W}}, v_{\mathrm{E}}\right)$. Denote the new exterior vertices by $v_{\mathrm{S}}$ and the resulting plane graph by $G^{\prime}$. (See Fig. 7 for an example.) $G^{\prime}$ does not have separating triangles, and it has four exterior vertices $v_{\mathrm{W}}, v_{\mathrm{S}}, v_{\mathrm{E}}, v_{\mathrm{N}}$ in ccw order on its own exterior face. Thus, $G^{\prime}$ is a PTP graph and has an REL $\left(S_{1}, S_{2}\right)$ according to Lemma 10.

We investigate the properties of the REL of $G^{\prime}$. Denote by $G_{1}$ the directed subgraph of $G^{\prime}$ induced by $S_{1}$ and the four exterior edges directed as $v_{\mathrm{S}} \rightarrow v_{\mathrm{W}}, v_{\mathrm{W}} \rightarrow v_{\mathrm{N}}, v_{\mathrm{S}} \rightarrow$ $v_{\mathrm{E}}, v_{\mathrm{E}} \rightarrow v_{\mathrm{N}}$. Let $E_{1}$ be the edge set of $G_{1}$. ( $E_{1}$ is the union of $S_{1}$ and the four exterior edges.) Then $G_{1}$ is an $s t$-plane graph with source $v_{\mathrm{S}}$ and sink $v_{\mathrm{N}}$. Similarly, let $G_{2}$ be the directed subgraph of $G^{\prime}$ induced by $S_{2}$ and the four exterior edges directed as $v_{\mathrm{W}} \rightarrow v_{\mathrm{S}}, v_{\mathrm{S}} \rightarrow v_{\mathrm{E}}, v_{\mathrm{W}} \rightarrow v_{\mathrm{N}}, v_{\mathrm{N}} \rightarrow v_{\mathrm{E}}$. Let $E_{2}$ be the edge set of $G_{2}$. Then $G_{2}$ is an $s t$-plane graph with source $v_{\mathrm{W}}$ and sink $v_{\mathrm{E}}$. We call $G_{1}$ the S-N net and $G_{2}$ the W-E net of $G^{\prime}$ derived from the REL $\left(S_{1}, S_{2}\right)$. For example, Fig. 8 shows an REL with its derived S-N and W-E nets for the PTP graph $G^{\prime}$ shown in Fig. 7. (Ignore the small boxes containing integers for now. Their meaning will be explained later.)

Consider the S-N net $G_{1}$. For each edge $e \in E_{1}$, let left $(e)(\operatorname{right}(e)$, resp.) denote the face of $G_{1}$ on the left (right, resp.) of $e$. Define the dual graph of $G_{1}$, denoted by $G_{1}^{*}$ as follows. The node set of $G_{1}^{*}$ is the set of the interior faces of $G_{1}$ plus two exterior faces $f_{\mathrm{W}}$ and $f_{\mathrm{E}}$ (which are obtained by "splitting" the exterior face of $G_{1}$ ). For each edge $e \in E_{1}$, there is a corresponding edge $e^{*}$ in $G_{1}^{*}$ directed from the face left $(e)$ to the face $\operatorname{right}(e) . G_{1}$ is an $s t$-plane graph, so $G_{1}^{*}$ is also an $s t$-plane graph with $f_{\mathrm{W}}$ as the only source and $f_{\mathrm{E}}$ as the only sink [21], [26]. For each face $f$ in $G_{1}$, define the upper left (upper right, resp.) edge of $f$ to be the last edge of the left (right, resp.) path of $f$, the lower left (lower right, resp.) edge of $f$ to be the first edge of the left (right, resp.) path of $f$. Note that these four edges are distinct. (This is because $\left(S_{1}, S_{2}\right)$ is an REL, so each of the two directed paths on the boundary of $f$ contains at least two edges.) 


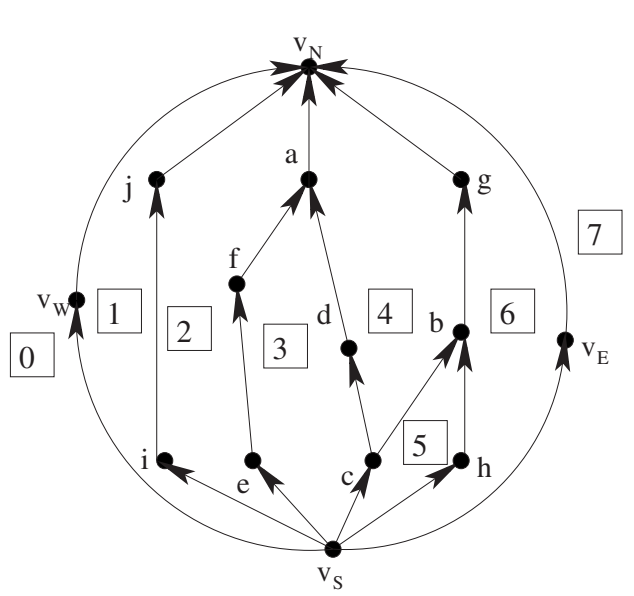

(a)

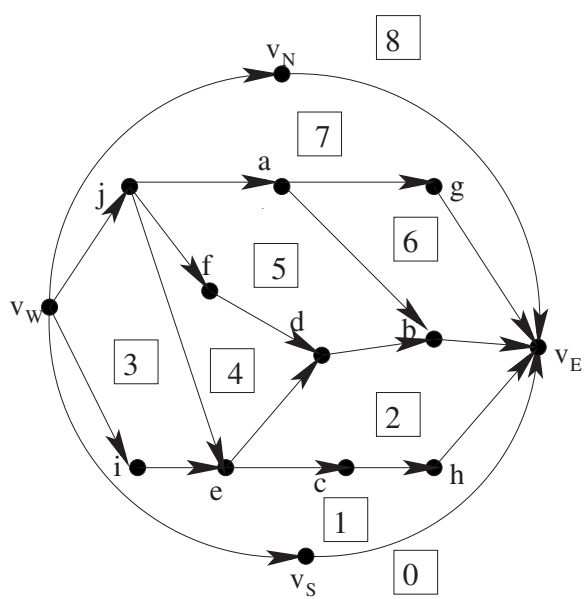

(b)

Fig. 8. (a) The S-N net $G_{1}$ and (b) the W-E net $G_{2}$ for the PTP graph $G^{\prime}$ in Fig. 7.

Similarly, we can define the dual $G_{2}^{*}$ for $G_{2}$ with the word "left" ("right", resp.) replaced by the word "below" ("above", resp.). $G_{2}^{*}$ is also an $s t$-plane graph with source $f_{\mathrm{S}}$ and sink $f_{\mathrm{N}}$. For each face $f$ in $G_{2}$, we similarly define the left above, left below, right above, and right below edge of $f$. These four edges are also distinct.

For $i=1,2$, assume $G_{i}$ has $k_{i}$ faces. Thus $G_{i}^{*}$ has $k_{i}+1$ nodes. By using topological sort, we can assign a distinct number from $0,1, \ldots, k_{1}$ to each node of $G_{1}^{*}$ such that, for any edge $e$ in $E_{1}$, the number assigned to the face left $(e)$ is smaller than the number assigned to the face right $(e)$. The faces $f_{\mathrm{W}}$ and $f_{\mathrm{E}}$ are numbered by 0 and $k_{1}$, respectively. Similarly, we can assign a distinct number from $0,1, \ldots, k_{2}$ to each node of $G_{2}^{*}$ such that, for any edge $e$ in $E_{2}$, the number assigned to the face below $(e)$ is smaller than the number assigned to the face above $(e)$. Such an assignment is called a consistent numbering of $G_{i}, i=1,2$ [12]. They can be computed in linear time by using topological sort.

Fix a consistent numbering of $G_{1}$. Let $f_{r}\left(0 \leq r \leq k_{1}\right)$ denote the face numbered by $r$. We define the ith $S-N$ separation path $S N_{i}$ to be the directed path in $G_{1}$ such that the faces numbered by $0,1, \ldots, i-1$ are on its left and the other faces are on its right. (These paths were called the path system in [12].) $G_{1}$ has exactly $k_{1} \mathrm{~S}-\mathrm{N}$ separation paths and all of them are directed from $v_{\mathrm{S}}$ to $v_{\mathrm{N}}$. Note that $S N_{r+1}$ is obtained from $S N_{r}$ by deleting the left path of the face $f_{r}$ and adding the right path of $f_{r}$.

The $j$ th $W-E$ separation path $W E_{j}$ of $G_{2}$ is defined similarly. $G_{2}$ has exactly $k_{2} \mathrm{~W}-\mathrm{E}$ separation paths and all of them are directed paths from $v_{\mathrm{W}}$ to $v_{\mathrm{E}}$ [12].

For example, the faces in Fig. 8 are consistently numbered for $G_{1}$ and $G_{2}$ (indicated by the integers in small boxes). The fourth W-E separation path $W E_{4}$ in $G_{2}$ consists of the vertices $v_{\mathrm{W}}, j, e, d, b, v_{\mathrm{E}}$. Note that there are four vertices $\left(i, c, h, v_{\mathrm{S}}\right)$ below it and four vertices $\left(f, a, g, v_{\mathrm{N}}\right)$ above it. 
In order to prove our main theorem, we need the following technical lemma:

Lemma 11. Let $G^{\prime}$ be a PTP graph with $n$ vertices. Let $G_{1}$ be the $S-N$ net and let $G_{2}$ be the $W-E$ net derived from an REL $\left(S_{1}, S_{2}\right)$ of $G^{\prime}$. Suppose $G_{1}$ has $k_{1}$ faces and $G_{2}$ has $k_{2}$ faces. Then:

(1) $k_{1}+k_{2}=n+1$.

(2) The $i$ th $S-N$ separation path $S N_{i}$ has at least $i-1$ vertices on its left and at least $k_{1}-i$ vertices on its right in $G_{1}$. The $j$ th $W-E$ separation path $W E_{j}$ has at least $j-1$ vertices below it and at least $k_{2}-j$ vertices above it in $G_{2}$.

Proof. (1) We apply Euler's formula to the graph $G_{1}$ and $G_{2}$. Because $G_{1}, G_{2}$ have the same vertex set as $G$, we have $|V|+k_{1}-2=\left|E_{1}\right|$ and $|V|+k_{2}-2=\left|E_{2}\right|$. Adding these two identities, we have $2|V|+k_{1}+k_{2}-4=\left|E_{1}\right|+\left|E_{2}\right|$. Since $E_{1}$ and $E_{2}$ double use four exterior edges, we have $\left|E_{1}\right|+\left|E_{2}\right|-4=3 n-7$. So $k_{1}+k_{2}=n+1$.

(2) We prove that there are at least $i-1$ vertices on the left of $S N_{i}$. The statement that there are at least $k_{1}-i$ vertices on the right of $S N_{i}$ can be proved by symmetry.

Denote the set of the vertices on the left of $S N_{i}$ by $S_{i}$. We show that $\left|S_{i}\right| \geq i-1$ by induction. When $i=1$, it is trivially true. Let us assume it is true when $i=r$, which means $\left|S_{r}\right| \geq r-1$. Consider $S_{r+1}$. The only face of $G_{1}$ enclosed by paths $S N_{r}$ and $S N_{r+1}$ is the face $f_{r}$ numbered by $r$. The left path $P$ of the face $f_{r}$ is on $S N_{r}$, but not on $S N_{r+1} . P$ contains at least one internal vertex $v$ that is neither the source nor the sink of $f_{r}$. Since $v$ is on $S N_{r}$, it is not on the left of $S N_{r}$. So $v \notin S_{r}$. However, $v \in S_{r+1}$. Thus $S_{r+1}$ contains $S_{r}$ plus at least one extra vertex $v$. By induction hypotheses, $\left|S_{r}\right| \geq r-1$. So $\left|S_{r+1}\right| \geq r$. This completes the induction.

The statement for the W-E separation paths can be proved similarly.

For example, in Fig. 8(a), when we expand from $S_{3}$ to $S_{4}$, the left path $P$ of the face numbered 3 is the path $\left(v_{\mathrm{S}}, e, f, a\right)$. The internal vertices $e, f$ of $P$ are not in $S_{3}$, but they are in $S_{4}$.

Next, we show how to get a canonical ordering tree from the S-N net and the W-E net, respectively. We prove that one of the two canonical ordering trees has at most $\lceil(n+1) / 2\rceil$ leaves.

Theorem 5. Let $G$ be a 4-connected plane triangulation with three exterior vertices $v_{\mathrm{W}}, v_{\mathrm{E}}, v_{\mathrm{N}}$ in ccw order. Delete the edge $\left(v_{\mathrm{W}}, v_{\mathrm{E}}\right)$. Denote the new exterior vertex by $v_{\mathrm{S}}$ and the resulting graph by $G^{\prime}$. Let $\left(S_{1}, S_{2}\right)$ be an REL of $G^{\prime}$. Let $G_{1}$ be the $S-N$ net and let $G_{2}$ be the $W-E$ net derived from $\left(S_{1}, S_{2}\right)$. Then the following statements hold:

(1) For each vertex $v \neq v_{\mathrm{N}}, v_{\mathrm{S}}$ in $G_{1}$, select an outgoing edge in $G_{1}$. For $v_{\mathrm{S}}$, select an outgoing edge not leading to $v_{\mathrm{W}}$ or $v_{\mathrm{E}}$. Then the set $T_{1}$ of the selected edges is a canonical ordering tree of $G$.

(2) For each vertex $v \neq v_{\mathrm{W}}, v_{\mathrm{E}}$ in $G_{2}$, select an outgoing edge in $G_{2}$. For $v_{\mathrm{W}}$, select the edge $\left(v_{\mathrm{W}}, v_{\mathrm{E}}\right)$. Then the set $T_{2}$ of the selected edges is a canonical ordering tree of $G$.

(3) One of $T_{1}, T_{2}$ has at most $\lceil(n+1) / 2\rceil$ leaves. It can be obtained in linear time. 


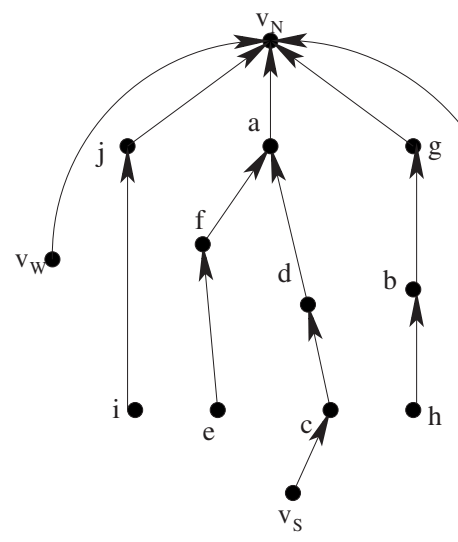

(a)

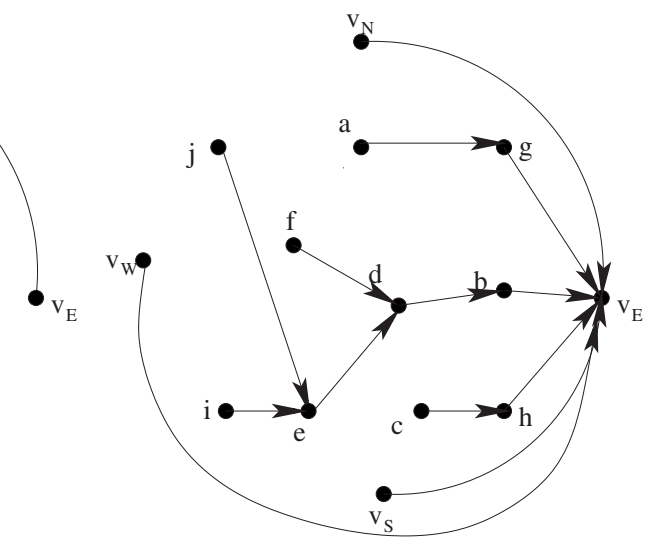

(b)

Fig. 9. (a) A canonical ordering tree of $G$ from the S-N net in Fig. 8(a). (b) A canonical ordering tree of $G$ from the W-E net in Fig. 8(b).

Proof. Figure 9 (a) shows the tree described in statement (1) for the S-N net in Fig. 8(a). Figure 9(b) shows the tree described in statement (2) for the W-E net in Fig. 8(b).

(1) Since $G_{1}$ is an acyclic digraph, $T_{1}$ is acyclic and each vertex, except $v_{\mathrm{N}}$, has exactly one outgoing edge in $T_{1}$. So $T_{1}$ is a directed tree rooted at $v_{\mathrm{N}}$.

Claim 1. All edges in $G-T_{1}$ are unrelated with respect to $T_{1}$.

Suppose, on the contrary, there exists an edge $e=\left(v_{j}, v_{i}\right)$ in $G-T_{1}$ that is related with respect to $T_{1}$. Without loss of generality, suppose $v_{i}$ is an ancestor of $v_{j}$ in $T_{1}$. Let $P$ be the unique path in $T_{1}$ from $v_{j}$ to $v_{i}$. Because the edge set $E$ of $G$ is the union of three disjoint edge subsets $S_{1}, S_{2}$ and $\left\{\left(v_{\mathrm{W}}, v_{\mathrm{S}}\right),\left(v_{\mathrm{S}}, v_{\mathrm{E}}\right),\left(v_{\mathrm{W}}, v_{\mathrm{N}}\right),\left(v_{\mathrm{E}}, v_{\mathrm{N}}\right),\left(v_{\mathrm{W}}, v_{\mathrm{E}}\right)\right\}$, there are three possible cases.

Case 1: $e$ is in $S_{1}$. Because there is no directed cycle in $G_{1}, e$ is directed from $v_{j}$ to $v_{i}$ in $G_{1}$. Let $R$ be the region in $G_{1}$ enclosed by the path $P$ and the edge $e$. Let $f$ be the face of $G_{1}$ that is inside $R$ and contains $e$ on its boundary. Then $v_{j}$ and $v_{i}$ are the source and the sink of $f$, respectively. Thus $f$ has one side consisting of only one edge $e=\left(v_{j}, v_{i}\right)$. This is impossible.

Case 2: $e$ is in $S_{2}$. Then $e$ is inside an interior face $f$ of $G_{1}$. So the path $P$ is on one side of $f$. Without loss of generality, assume $P$ is on the left side of $f$. If $v_{i}$ is the sink of $f$, then there is another path $P^{\prime}$ on the right side of $f$ ending at $v_{i}$. Thus, $e \in S_{2}$ is sandwiched between two incoming edges of $G_{1}$ incident to $v_{i}$. This is impossible. Similarly, $v_{j}$ cannot be the source of $f$. Thus $v_{j}$ and $v_{i}$ are internal vertices of the left path of $f$. From the definition of REL, the edge $e=\left(v_{j}, v_{i}\right)$ must be directed as outgoing from $v_{j}$ and outgoing from $v_{i}$ in $G_{2}$. This is impossible.

Case 3: $e$ is in $\left\{\left(v_{\mathrm{W}}, v_{\mathrm{S}}\right),\left(v_{\mathrm{S}}, v_{\mathrm{E}}\right),\left(v_{\mathrm{W}}, v_{\mathrm{N}}\right),\left(v_{\mathrm{E}}, v_{\mathrm{N}}\right),\left(v_{\mathrm{W}}, v_{\mathrm{E}}\right)\right\} . \quad\left(v_{\mathrm{W}}, v_{\mathrm{N}}\right)$ and $\left(v_{\mathrm{E}}, v_{\mathrm{N}}\right)$ are in $T_{1}$, so $e \neq\left(v_{\mathrm{W}}, v_{\mathrm{N}}\right)$ and $e \neq\left(v_{\mathrm{E}}, v_{\mathrm{N}}\right)$. Thus $e$ has to be one of $\left(v_{\mathrm{W}}, v_{\mathrm{E}}\right),\left(v_{\mathrm{W}}, v_{\mathrm{S}}\right)$ 


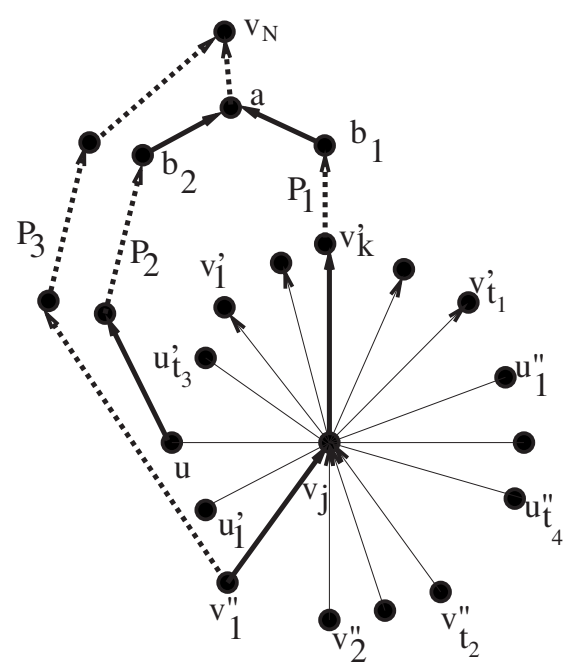

Fig. 10. The vertex $v_{j}$ is orderly in tree $T_{1}$.

or $\left(v_{\mathrm{S}}, v_{\mathrm{E}}\right)$. Both ending vertices of these three edges are leaves of $T_{1}$, none of them can be an ancestor of the other. So $e$ cannot be related with respect to $T_{1}$. Thus this case is also impossible.

This completes the proof of the claim. It remains to show that every vertex $v$ of $G$ is orderly with respect to $T_{1}$.

Let $v_{1}, v_{2}, \ldots, v_{n}$ be the ccw preordering of the vertices of $G$ with respect to $T_{1}$. Obviously, $v_{\mathrm{N}}, v_{\mathrm{W}}, v_{\mathrm{E}}$ are numbered $1,2, n$, respectively (see Fig. 9(a)). It is easy to verify that they are orderly with respect to $T_{1}$.

Consider any other vertex $v_{j}, j \neq 1,2, n$, in $T_{1}$. Let $\left(v_{j}, v_{1}^{\prime}\right), \ldots,\left(v_{j}, v_{t_{1}}^{\prime}\right)$ be the outgoing edges from $v_{j}$ in cw order in $S_{1}$; let $\left(v_{1}^{\prime \prime}, v_{j}\right),\left(v_{2}^{\prime \prime}, v_{j}\right), \ldots,\left(v_{t_{2}}^{\prime \prime}, v_{j}\right)$ be the incoming edges to $v_{j}$ in ccw order in $S_{1} ;\left(u_{1}^{\prime}, v_{j}\right), \ldots,\left(u_{t_{3}}^{\prime}, v_{j}\right)$ be the incoming edges to $v_{j}$ in cw order in $S_{2}$; and let $\left(v_{j}, u_{1}^{\prime \prime}\right), \ldots,\left(v_{j}, u_{t_{4}}^{\prime \prime}\right)$ be the outgoing edges from $v_{j}$ in cw order in $S_{2}$. Suppose the edge $\left(v_{j}, v_{k}^{\prime}\right)$ is selected into $T_{1}$. (See Fig. 10 for an illustration. The edges in $S_{1}$ are arrowed. The edges in $S_{2}$ are not arrowed.)

First, observe that each vertex $v_{p}^{\prime \prime}\left(2 \leq p \leq t_{2}-1\right)$ has only one outgoing edge $\left(v_{p}^{\prime \prime}, v_{j}\right)$ in $S_{1}$. Hence all edges $\left(v_{p}^{\prime \prime}, v_{j}\right)\left(2 \leq p \leq t_{2}-1\right)$ must be selected and included in $T_{1}$.

If $\left(v_{1}^{\prime \prime}, v_{j}\right)$ is in $T_{1}$, let $B_{2}\left(v_{j}\right)=\left\{u_{1}^{\prime}, \ldots, u_{t_{3}}^{\prime}, v_{1}^{\prime}, \ldots, v_{k-1}^{\prime}\right\}$. Otherwise, if $\left(v_{1}^{\prime \prime}, v_{j}\right)$ is not in $T_{1}$, let $B_{2}\left(v_{j}\right)=\left\{v_{1}^{\prime \prime}, u_{1}^{\prime}, \ldots, u_{t_{3}}^{\prime}, v_{1}^{\prime}, \ldots, v_{k-1}^{\prime}\right\} . B_{2}\left(v_{j}\right)$ is a consecutive block of neighbors of $v_{j}$. We want to show that the ccw preordering number of every vertex in $B_{2}\left(v_{j}\right)$ is smaller than $j$. Let $P_{1}$ be the unique path in $T_{1}$ connecting $v_{j}$ to the root $v_{\mathrm{N}}$. Consider a vertex $u$ in $B_{2}\left(v_{j}\right)$. Let $P_{2}$ be the unique path in $T_{1}$ from $u$ to $v_{\mathrm{N}}$. Let $a$ be the lowest common vertex of $P_{1}$ and $P_{2}$. Let $b_{1}$ and $b_{2}$ be the children of $a$ that are on $P_{1}$ and $P_{2}$, respectively. Starting at $v_{1}^{\prime \prime}$, we trace a unique path $P_{3}$ in $G_{1}$ as follows: for each vertex $v$ we travel to its leftmost outgoing edge in $G_{1}$. Continue this until reaching $v_{\mathrm{N}}$. 
Let $R$ be the closed region enclosed by the edge $\left(v_{1}^{\prime \prime}, v_{j}\right)$, the paths $P_{1}$ and $P_{3}$. Since the vertex $u$ is inside $R, P_{2}$ is inside $R$. Thus $P_{2}$ is on the left of $P_{1}$ and on the right of $P_{3}$. So the edge $\left(a, b_{2}\right)$ appears before the edge $\left(a, b_{1}\right)$ in ccw order around $a$. This implies that the ccw preordering number of $u$ with respect to $T_{1}$ is smaller than $j$ as was to be shown.

Similarly, we can define the vertex set $B_{4}\left(v_{j}\right)$ on the other side of $v_{j}$, and prove that the ccw preordering number of every vertex in $B_{4}\left(v_{j}\right)$ is bigger than $j$. Thus, $v_{j}$ is orderly with respect to $T_{1}$. Since this is true for any vertex $v_{j}, T_{1}$ is an orderly spanning tree of $G$, hence also a canonical ordering tree of $G$ by Lemma 2(1).

(2) The proof is similar to (1).

(3) Assume $G_{1}$ has $k_{1}$ faces and $G_{2}$ has $k_{2}$ faces. The canonical ordering tree $T_{1}$ can also be obtained by deleting edges from $G_{1}$. Because deleting one edge destroys one face of $G_{1}$ and $T_{1}$ is connected, the number of edges that must be deleted from $G_{1}$ to make $T_{1}$ is exactly the number of faces of $G_{1}$ minus 1 , namely, $k_{1}-1$. For each leaf $v \neq v_{\mathrm{S}}$ of $T_{1}$, all (at least one) incoming edges to $v$ in $G_{1}$ are deleted. So $T_{1}$ has at most $k_{1}-1$ leaves other than $v_{\mathrm{S}}$. Adding the leaf $v_{\mathrm{S}}$, the number of leaves of $T_{1}$ is at most $k_{1}$.

Similar argument also apply to the canonical ordering tree $T_{2}$. However, since we add the edge $\left(v_{\mathrm{W}}, v_{\mathrm{E}}\right)$ into $T_{2}$ which results in one more leaf, the number of leaves of $T_{2}$ is at most the number of faces of $G_{2}$ plus 1 , namely, $k_{2}+1$. The sum of the numbers of the leaves in $T_{1}$ and $T_{2}$ is thus at most $k_{1}+k_{2}+1=n+2$ by Lemma 11(1). Hence one of $T_{1}$ and $T_{2}$ has at most $\lfloor(n+2) / 2\rfloor=\lceil(n+1) / 2\rceil$ leaves.

An REL of $G^{\prime}$ can be obtained in $O(n)$ time [19]. Obviously a canonical ordering tree $T$ with at most $\lceil(n+1) / 2\rceil$ leaves can be obtained in $O(n)$ time from an REL by the construction above.

\section{Compact Visibility Representation of 4-Connected Plane Graphs}

In this section we use the $\mathrm{S}-\mathrm{N}$ and $\mathrm{W}-\mathrm{E}$ nets of 4-connected plane triangulations to prove that a 4-connected plane graph admits a VR with height at most $\lceil 3 n / 4\rceil$ :

Theorem 6. Every 4-connected plane graph $G$ with $n$ vertices has a VR with height at most $\lceil 3 n / 4\rceil$, which can be obtained in linear time.

Proof. Without loss of generality, assume $G$ is a 4-connected plane triangulation with three exterior vertices $v_{\mathrm{W}}, v_{\mathrm{E}}, v_{\mathrm{N}}$ in ccw order. Delete the edge $\left(v_{\mathrm{W}}, v_{\mathrm{E}}\right)$ and denote the new exterior vertex by $v_{\mathrm{S}}$ and the resulting PTP graph by $G^{\prime}$. Let $\left(S_{1}, S_{2}\right)$ be an REL of $G^{\prime}$. Let $G_{1}, G_{2}$ be the S-N and W-E nets derived from $\left(S_{1}, S_{2}\right)$. Assume $G_{1}$ has $k_{1}$ faces and $G_{2}$ has $k_{2}$ faces. By Lemma 11(1), $k_{1}+k_{2}=n+1$. Assign consistent numbers to faces of $G_{1}$ and $G_{2}$. There are two cases to consider:

Case 1: $k_{2} \geq(n+1) / 2$. We use $G_{2}$ to construct a canonical ordering tree of $G$. Let $P$ be the $\left\lfloor\left(k_{2}+1\right) / 2\right\rfloor$ th W-E separation path. For the vertex $v_{\mathrm{W}}$, select the edge $\left(v_{\mathrm{W}}, v_{\mathrm{E}}\right)$. For each vertex on $P$ other than $v_{\mathrm{W}}$, select its outgoing edge in $P$. For any other vertex $v$, select one outgoing edge from $v$ in $G_{2}$. The set of selected edges is an orderly spanning tree $T$ of $G$ by Theorem 5(2) and Lemma 2(1). With respect to the ccw preordering of $T$, every interior vertex $v$ has nonempty neighbor sets $B_{2}(v)$ and $B_{4}(v)$. 


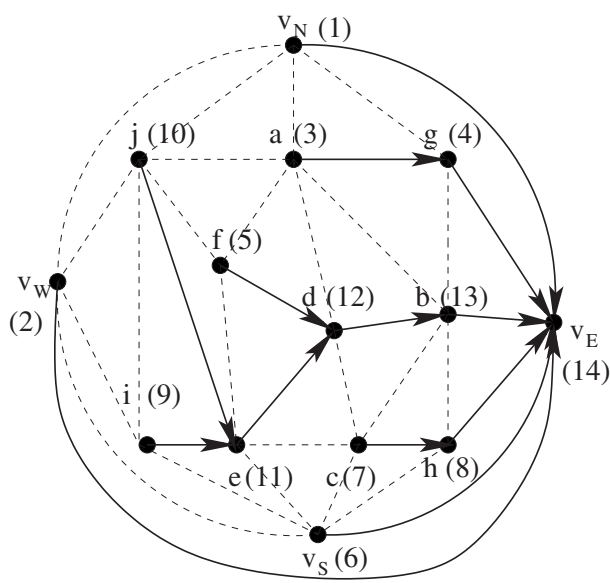

(a)

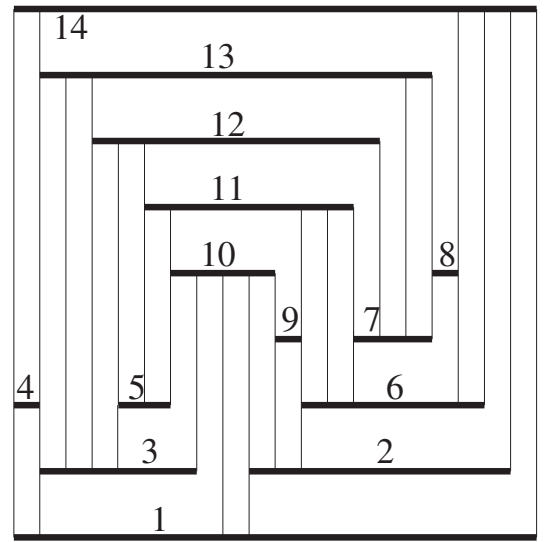

(b)

Fig. 11. An $s t$-numbering and its corresponding VR of graph $G$ in Fig. 7.

Next, we construct an $s t$-numbering of $G$ using $T$. We preassign 2 to $v_{\mathrm{W}}$. Then we assign numbers $1,3, \ldots, n$ to other vertices of $G$ in the ccw postordering of $T$, starting from the vertex $v_{\mathrm{N}}$ until we reach the vertices on $P$. Do not assign numbers to vertices on $P$ at this moment. Then we jump to the vertex $v_{\mathrm{S}}$ and assign the remaining numbers in the cw postordering of $T$, starting from $v_{\mathrm{S}}$, until we reach the vertices on $P$. Keep assigning the remaining numbers to the vertices on $P$ until reaching the root $v_{E}$, which is assigned $n$.

Figure 11(a) shows an example. We choose the W-E net $G_{2}$ in Fig. 8(b) and the fourth W-E separation path $\left(v_{\mathrm{W}}, j, e, d, b, v_{\mathrm{E}}\right)$. The edges of the chosen canonical ordering tree are drawn in solid lines. Other edges are drawn in dashed lines. The resulting stnumbering is shown in integers in the parentheses.

Let $R_{1}$ denote the closed region enclosed by $P$ and the edge $\left(v_{\mathrm{W}}, v_{\mathrm{E}}\right)$. Let $R_{2}$ denote the closed region enclosed by $P$ and the path $\left(v_{\mathrm{W}}, v_{\mathrm{N}}, v_{\mathrm{E}}\right)$.

We now show that this numbering is indeed an $s t$-numbering of $G$. For any vertex $v$ other than the root $v_{\mathrm{E}}$, its parent in $T$ is a bigger neighbor of $v$. Thus we only need to show that each vertex $v \neq v_{\mathrm{N}}$ has at least one smaller neighbor. There are several cases:

(1) $v=v_{\mathrm{W}}: v_{\mathrm{N}}$ is a smaller neighbor of $v$.

(2) $v=v_{\mathrm{S}}: v_{\mathrm{W}}$ is a smaller neighbor of $v$.

(3) $v=v_{\mathrm{E}}$ : Every neighbor is a smaller neighbor of $v$.

(4) $v$ is inside $R_{2}: v$ is an interior vertex of $G$. Thus all the vertices in the nonempty set $B_{2}(v)$ are assigned smaller numbers than $v$.

(5) $v \neq v_{\mathrm{S}}$ is inside $R_{1}: v$ is an interior vertex of $G$. Thus all the vertices in the nonempty set $B_{4}(v)$ are assigned smaller numbers than $v$.

(6) $v$ is an internal vertex of the path $P$ : All the vertices in the nonempty sets $B_{2}(v)$ and $B_{4}(v)$ are assigned smaller numbers than $v$.

Therefore this numbering is indeed an $s t$-numbering of $G$. 
Consider any directed path $P^{\prime}$ in the resulting $s t$-orientation. If $P^{\prime}$ passes through a vertex $u$ inside $R_{1}$ and a vertex $v$ inside $R_{2}$, then $P^{\prime}$ has to intersect the path $P$ at a vertex $v^{\prime}$, which should be numbered between $u$ and $v$. However, for any vertex $v^{\prime}$ on $P$, either $v^{\prime}=v_{\mathrm{W}}$, then it is numbered smaller than both $u$ and $v$, or $v^{\prime} \neq v_{\mathrm{W}}$, then it is numbered bigger than both $u$ and $v$. So this is impossible. Thus, $P^{\prime}$ has to bypass either all vertices inside $R_{1}$ or all vertices inside $R_{2}$. Therefore it has to bypass at least $\lfloor n / 4\rfloor$ vertices by Lemma 11(2). So the length of any directed path is at most $\lceil 3 n / 4\rceil$.

Applying the VR algorithm in Lemma 1 to this $s t$-numbering gives a VR of $G$ with height at most $\lceil 3 n / 4\rceil$. It can be obtained in $O(n)$ time.

Figure 11(b) shows a VR of the graph $G$ using the $s t$-numbering in Fig. 11(a).

Case 2: $k_{1} \geq(n+1) / 2$. It is similar to Case 1 , except that, instead of using $G_{2}$, we use $G_{1}$ to construct the required $s t$-numbering of $G$.

We know that the S-N and W-E nets can be obtained in linear time. Obviously, the $s t$-numbering of $G$ constructed above can be obtained from the S-N and W-E nets in linear time. Applying Lemma 1, the total running time of constructing the VR is linear.

\section{Conclusion and Open Problems}

In this paper we presented a new method to construct an $s t$-numbering of a plane triangulation $G$ by picking one canonical ordering tree from the minimum realizer of $G$. This guarantees that the length of the longest path in the resulting $s t$-orientation is at most $\lceil 15 n / 16\rceil$. This results in a VR of $G$ with height at most $\lceil 15 n / 16\rceil$. It deserves further study to see whether this bound can be reduced.

One interesting aspect is that our method prefers a canonical ordering tree with more leaves, which is contrary to most applications of canonical ordering trees. We proved that every plane triangulation has a canonical ordering tree with at least $\lceil(n+1) / 2\rceil$ leaves. It deserves further study to improve this bound.

We also gave a more compact straight-line grid embedding of plane graphs, also by using the minimum realizer. It is interesting to see if the bound can be further reduced.

We obtained a canonical ordering tree for a 4-connected plane triangulation with at most $\lceil(n+1) / 2\rceil$ leaves. An interesting problem deserving further consideration is whether there is a 4-connected plane triangulation $G$ such that every canonical ordering tree of $G$ has at least this many leaves.

We also showed that every 4-connected plane graph has a VR with height at most $\lceil 3 n / 4\rceil$. It deserves further study to improve this bound.

\section{References}

1. J. Bhasker and S. Sahni, A linear time algorithm to check for the existence of a rectangular dual of a planar triangulated graph, Networks 17 (1987), 307-317.

2. N. Bonichon, B. L. Saëc and M. De la Libération, Wagner's theorem on realizers, in Proc. 29th International Colloquium on Automata, Languages and Programming, pp. 1043-1053, Lecture Notes in Computer Science, Vol. 2380, Springer-Verlag, Berlin, 2002. 
3. E. Brehm, 3-Orientations and Schnyder 3-tree-decompositions, Diploma Thesis, FB Mathematik und Informatik, Freie Universität Berlin, 2000.

4. H.-L. Chen, C.-C. Liao, H.-I. Lu and H.-C. Yen, Some applications of orderly spanning trees in graph drawing, in Proc. Graph Drawing '02, pp. 332-343, Lecture Notes in Computer Science, Vol. 2528, Springer-Verlag, Berlin, 2002.

5. Y.-T. Chiang, C.-C. Lin and H.-I. Lu, Orderly spanning trees with applications to graph encoding and graph drawing, in Proc. 12th Annual ACM-SIAM SODA, pp. 506-515, ACM Press, New York, 2001.

6. M. Chrobak and G. Kant, Convex grid drawings of 3-connected planar graphs, Technical Report RUUCS-93-45, Department of Computer Science, Utrecht University, 1993.

7. M. Chrobak and T. Payne, A linear-time algorithm for drawing a planar graph on a grid, Inform. Process. Lett. 54 (1995), 241-246.

8. U. Fößmeier, G. Kant and M. Kaufmann, 2-Visibility drawings of planar graphs, in Proc. 4th International Symposium on Graph Drawing, pp. 155-168, Lecture Notes in Computer Science, Vol. 1190, SpringerVerlag, Berlin, 1996.

9. H. de Fraysseix and P. Ossona de Mendez, On topological aspects of orientations, Discrete Math. 229 (1-3) (2001), 57-72.

10. H. de Fraysseix, J. Pach and R. Pollack, Small sets supporting straight-line embeddings of planar graphs, in Proc. 20th Annual Symposium on Theory of Computing, pp. 426-433, ACM Press, New York, 1988.

11. H. de Fraysseix, J. Pach and R. Pollack, How to draw a planar graph on a grid, Combinatorica 10 (1990), $41-51$.

12. X. He, On finding the rectangular duals of planar triangular graphs, SIAM J. Comput. 22 (1993), 12181226.

13. X. He, Grid embedding of 4-connected plane graphs, Discrete Comput. Geom. 17 (1997), 339-358.

14. X. He, On floor-plan of plane graphs, SIAM J. Comput. 28 (1999), 2150-2167.

15. X. He, M.-Y. Kao and H.-I. Lu, Linear-time succinct encodings of planar graphs via canonical orderings, SIAM J. Discrete Math. 12 (1999), 317-325.

16. G. Kant, Drawing planar graphs using the 1mc-ordering, in Proc. 33rd Symposium on Foundations of Computer Science, pp. 101-110, IEEE, Pittsburgh, PA, 1992.

17. G. Kant, Algorithms for drawing planar graphs, Ph.D. Dissertation, Department of Computer Science, University of Utrecht, 1993.

18. G. Kant, A more compact visibility representation, Internat. J. Comput. Geom. Appl. 7 (1997), 197-210.

19. G. Kant and X. He, Regular edge labeling of 4-connected plane graphs and its applications in graph drawing problems, Theoret. Comput. Sci. 172 (1997), 175-193.

20. K. Koźmiński and E. Kinnen, Rectangular dual of planar graphs, Networks 15 (1985), 145-157.

21. A. Lempel, S. Even and I. Cederbaum, An algorithm for planarity testing of graphs, in Theory of Graphs (Proc. of an International Symposium, Rome, July 1966), pp. 215-232, 1967.

22. C.-C. Liao, H.-I. Lu and H.-C. Yen, Floor-planning via orderly spanning trees, in Proc. 9th International Symposium on Graph Drawing, pp. 367-377, Lecture Notes in Computer Science, Vol. 2265, SpringerVerlag, Berlin, 2002.

23. C.-C. Lin, H.-I. Lu and I.-F. Sun, Improved compact visibility representation of planar graph via Schnyder's realizer, in Proc. 20th Annual Symposium on Theoretical Aspects of Computer Science, pp. 14-25, Lecture Notes in Computer Science, Vol. 2607, Springer-Verlag, Berlin, 2003.

24. K. Miura, S. Nakano and T. Nishizeki, Grid drawings of 4-connected plane graphs, Discrete Comput. Geom. 26 (2001), 73-87.

25. P. Ossona de Mendez, Orientations bipolaires, Ph.D. thesis, Ecole des Hautes Etudes en Sciences Sociales, Paris, 1994.

26. P. Rosenstiehl and R. E. Tarjan, Rectilinear planar layouts and bipolar orientations of planar graphs, Discrete Comput. Geom. 1 (1985), 343-353.

27. W. Schnyder, Planar graphs and poset dimension, Order 5 (1989), 323-343.

28. W. Schnyder, Embedding planar graphs on the grid, in Proc. First Annual ACM-SIAM Symposium on Discrete Algorithms, pp. 138-148, SIAM, Philadelphia, PA, 1990.

29. R. Tamassia and I. G.Tollis, An unified approach to visibility representations of planar graphs, Discrete Comput. Geom. 1 (1986), 321-341.

Received May 29, 2003, and in revised form August 10, 2004. Online publication January 14, 2005. 